Draft version August 30, 2021

Typeset using $\mathrm{IAT}_{\mathrm{E} X} \mathrm{manuscript}$ style in AASTeX63

\title{
Whistler-regulated MHD: Transport equations for electron thermal conduction in the high $\beta$ intracluster medium of galaxy clusters
}

\author{
J. F. Drake, ${ }^{1,2,3,4}$ C. Pfrommer,${ }^{5}$ C. S. Reynolds, ${ }^{6}$ M. Ruszkowski,${ }^{7}$ M. Swisdak $, 3,4$ \\ A. Einarsson, ${ }^{1}$ T. Thomas,${ }^{5,8}$ A. B. Hassam, ${ }^{1,3}$ and G. T. Roberg-Clark ${ }^{9}$ \\ ${ }^{1}$ Department of Physics, University of Maryland, College Park, MD 20742, USA \\ ${ }^{2}$ Institute for Physical Science and Technology, University of Maryland, College Park, MD 20742, USA \\ ${ }^{3}$ Institute for Research in Electronics and Applied Physics, University of Maryland, College Park, MD 20742, USA \\ ${ }^{4}$ Joint Space-Science Institute (JSI), College Park, MD 20742, USA \\ ${ }^{5}$ Leibniz Institute for Astrophysics, Potsdam (AIP), An der Sternwarte 16, 14482 Potsdam, Germany \\ ${ }^{6}$ Institute of Astronomy, University of Cambridge, Cambridge CB3 OHA, UK \\ ${ }^{7}$ Department of Astronomy, University of Michigan, Ann Arbor, MI 48109, USA \\ ${ }^{8}$ Institute of Physics and Astronomy, University of Potsdam, Karl-Liebknecht-Str. 24/25, 14476 Golm, Germany \\ ${ }^{9}$ Max-Planck-Institute for Plasmaphysics, 17491 Greifswald, Germany
}

(Received August 30, 2021)

Submitted to ApJ

\begin{abstract}
Transport equations for electron thermal energy in the high $\beta_{e}$ intracluster medium (ICM) are developed that include scattering from both classical collisions and selfgenerated whistler waves. The calculation employs an expansion of the kinetic electron equation along the ambient magnetic field in the limit of strong scattering and assumes whistler waves with low phase speeds $V_{w} \sim v_{t e} / \beta_{e} \ll v_{t e}$ dominate the turbulent spec-
\end{abstract}

Corresponding author: James F. Drake drake@umd.edu 
trum, with $v_{t e}$ the electron thermal speed and $\beta_{e} \gg 1$ the ratio of electron thermal to magnetic pressure. We find: (1) temperature-gradient-driven whistlers dominate classical scattering when $L_{c}>L / \beta_{e}$, with $L_{c}$ the classical electron mean-free-path and $L$ the electron temperature scale length, and (2) in the whistler dominated regime the electron thermal flux is controlled by both advection at $V_{w}$ and a comparable diffusive term. The findings suggest whistlers limit electron heat flux over large regions of the ICM, including locations unstable to isobaric condensation. Consequences include: (1) the Field length decreases, extending the domain of thermal instability to smaller lengthscales, (2) the heat flux temperature dependence changes from $T_{e}^{7 / 2} / L$ to $V_{w} n T_{e} \sim T_{e}^{1 / 2},(3)$ the magneto-thermal and heat-flux driven buoyancy instabilities are impaired or completely inhibited, and (4) sound waves in the ICM propagate greater distances, as inferred from obserations. This description of thermal transport can be used in macroscale ICM models.

Keywords: conduction — galaxies: clusters: intracluster medium — methods: numerical — plasmas — turbulence

\section{INTRODUCTION}

Half of all baryons in the low-redshift Universe are in a hot phase, with temperatures in the range $T=10^{5-8} \mathrm{~K}$ (Macquart et al. 2020). Much of this matter exists as very diffuse plasma in the filaments of the cosmic web, heated by photoionization from stars and active galactic nuclei (AGN, McQuinn 2016) as well as electron-positron beam instabilities associated with TeV blazars (Broderick et al. 2012; Lamberts et al. 2015). Of more astrophysical significance are the hot baryons that have passed through cosmic accretion shocks (Ryu et al. 2003; Pfrommer et al. 2006; Schaal et al. 2016), which caused the baryons to virialize in the dark matter halos of massive $\left(>L^{*}\right)$ galaxies and galaxy clusters to form the hot circumgalactic medium (CGM) and hot intracluster medium (ICM), respectively.

The ICM is particularly well studied since it has typical temperatures $\left(T \sim 10^{7}-10^{8} \mathrm{~K}\right)$ and emission measures that make nearby galaxy clusters amongst the brightest objects in the extragalactic X-ray 
sky. We now understand that the ICM dominates the baryon budget of clusters (containing $80 \%$ of the baryons) and forms an approximately hydrostatic atmosphere in the dark matter potential. In relaxed clusters (i.e., those not undergoing major mergers), the core regions of the ICM develop short cooling times and, left unchecked, a cooling catastrophe would occur resulting in significant star formation events $\left(100-1000 \mathrm{M}_{\odot}\right)$ and bright central galaxies $(B C G s)$ with very massive $\left(10^{13} \mathrm{M}_{\odot}\right)$ stellar components (Sarazin 1986; Fabian 1994). The fact that BCGs are significantly less massive and never host such star forming events demonstrates that the ICM cooling must be balanced by a heat source (Peterson \& Fabian 2006). The current paradigm is that the jets from the central radio-loud active galactic nucleus (AGN) hosted by the BCG provide that heating (Churazov et al. 2000; Reynolds et al. 2002), although the precise mechanisms involved in thermalizing the AGN energy injection remain unclear (see discussion in Bambic \& Reynolds 2019). Thus, studies of galaxy clusters and the ICM give us a unique window on the AGN feedback processes that shape the most massive galaxies in the Universe. A key ingredient needed to disentangle these complex systems is the microphysics, and especially the transport properties, of the hot ICM.

Thermal transport in the ICM is particularly important (Binney \& Cowie 1981). Thermal conduction into the cooling ICM core from the hotter outer regions can reduce, but not eliminate, the need for AGN heating (Stewart et al. 1984; Bregman \& David 1988; Zakamska \& Narayan 2003; Voigt \& Fabian 2004). Thermal transport may be crucial in dissipating weak shocks and acoustic modes driven by the AGN (Fabian et al. 2005a; Zweibel et al. 2018) thereby thermalizing AGN injected energy. Thermal transport is also important for modifying the local thermal instabilities responsible for the condensation of cold gas that, ultimately, fuels the AGN (Yang \& Reynolds 2016). But despite its importance, the basic physics of thermal transport in ICM-like plasmas has yet to be fully understood. At typical densities $\left(n_{e} \sim 10^{-3}-10^{-1} \mathrm{~cm}^{-3}\right)$ and temperatures, the electron mean free path to ion scattering is $L_{c} \sim 0.1-1 \mathrm{kpc}$ and thus only 1-3 orders of magnitude smaller than global scales. The ICM is magnetized with typical fields $B \sim 1-10 \mu \mathrm{G}$, giving rise to electron gyro-radii $\rho_{e} \sim 0.1-1 \mathrm{npc}$ many orders of magnitude smaller than $\lambda$, implying that the transport will be highly suppressed in the cross-field direction and thus anisotropic. Most current treatments 
of the ICM adopt a fluid description and take the thermal transport parallel to the local magnetic field to be described by the canonical theory of Spitzer (1956). However, the Spitzer ansatz assumes a high degree of electron-ion collisionality which is not obviously true for the ICM. The fact that the ratio of thermal-to-magnetic pressure is large $(\beta \sim 100)$ further raises the spectre of kinetic instabilities driven by pressure anisotropies and heat fluxes (Gary \& Li 2000; Schekochihin et al. 2005; Kunz et al. 2014; Rincon et al. 2015; Riquelme et al. 2016; Komarov et al. 2016).

More recently the focus has shifted to the role of whistler waves that are directly driven by electron heat flux in suppressing transport (Levinson \& Eichler 1992; Pistinner \& Eichler 1998; Roberg-Clark et al. 2016, 2018a; Komarov et al. 2018). Transport suppression is dominated by oblique waves that can resonate with whistlers propagating in the direction of the electron heat flux through the Landau $(n=0)$ and "anomalous" $(n=-1,-2, \ldots)$ resonances $\omega-k_{\|} v_{\|}-n \Omega_{e}=0$, where $\Omega_{e}=e B / m_{e} c$ is the electron cyclotron frequency. Particle-in-cell (PIC) simulations carried out in two dimensions revealed that the whistler fluctuations reach large amplitude and are dominated by wavenumbers with $k \rho_{e} \sim 1$, where $\rho_{e}=v_{t e} / \Omega_{e}$ is the electron Larmor radius. The dispersion relation for whistlers with wavelength $k d_{e} \sim d_{e} / \rho_{e} \ll 1$ is given by

$$
\omega=k_{\|} k d_{e}^{2} \Omega_{e} \ll \Omega_{e}
$$

with $d_{e}$ the collisionless skin depth. The parallel phase speed $V_{w}=\omega / k_{\|}$of whistlers in this regime therefore scales like $v_{t e} / \beta_{e} \ll v_{t e}$, where $\beta_{e}=8 \pi n T_{e} / B^{2}$ is the ratio of electron thermal to magnetic field pressure. Note that in the definition of $\beta_{e}$, and in the remainder of this paper, the electron temperature will be expressed in energy units. The simulations revealed that electron scattering was strong enough that hot electrons were constrained to move with the whistler phase speed down the temperature gradient, reducing the heat flux well below the free-streaming value $Q_{0} \sim n T_{e} v_{t e}$ to a level that scaled as $Q_{0} / \beta_{e}$ (Pistinner \& Eichler 1998; Roberg-Clark et al. 2018a; Komarov et al. 2018). Interestingly, the inverse scaling of the electron heat flux with $\beta_{e}$ has been documented in the solar wind (Tong et al. 2018, 2019). Moreover, recent observations from the Parker Solar Probe, revealed large amplitude, oblique whistler waves embedded in regions of depressed magnetic field 
intensity where the local $\beta_{e}$ was high (Agapitov et al. 2020; Cattell et al. 2021a). The waves were associated with the $n=-1$ anomalous resonance and scattered the field-aligned strahl electrons into the halo population with a broad range of pitch angles (Cattell et al. 2021b).

Due to computational constraints, PIC simulations exploring heat flux suppression employ ambient temperature gradients that are artificially large, with gradient scale lengths that are only hundreds of electron Larmor radii. Thus, while the simulations suggested that the scaling of the suppressed heat flux was insensitive to the ambient gradient, the extrapolation to physical systems requires that the results scale as expected over many orders of magnitude. Further, simulations to date have been carried out in the limit where classical collisions are negligible so that their impact on the onset of the whistler instability is uncertain. Thus, the transition from whistler-limited to classical-collisionlimited transport has not been explored beyond the construction of ad hoc connection formulas to bridge the two limits (Komarov et al. 2018).

The goal of the present work is to develop a set of transport equations that treat macroscale systems while simultaneously describing the transition from classical- to whistler-limited transport. Two characteristics of scattering by whistler waves make the calculation possible. First, whistlers scatter electrons on constant energy surfaces in the frame moving with the whistler phase speed $V_{w}$ along the ambient magnetic field. Hence, the whistler scattering can be formulated as a pitch angle scattering operator moving at $V_{w}$. Second, because $V_{w}=v_{t e} / \beta_{e} \ll v_{t e}$, the kinetic equation for electrons can be solved by an ordering in which $V_{w} / v_{t e} \ll 1$. At the same time, the total scattering rate, $\nu(v)=\nu_{w}(v)+\nu_{e i}(v)$ includes both classical and whistler scattering and is ordered so that the mean-free-path $L_{\nu}=v_{t e} / \nu \sim L / \beta_{e}$ is short compared with the ambient temperature scale length along the magnetic field, $L$. To simplify the calculation, the classical collisions are also treated by a simple pitch-angle scattering operator, which is, of course, valid for electron-ion but not for electronelectron collisions. The final transport equations for the electron thermal energy result from solving the kinetic equation for the electron distribution $f(x, v, \zeta, t)$ to second order in the small parameter $\epsilon \sim V_{w} / v_{t e} \sim L_{\nu} / L$ with no assumptions made about the relative sizes of the classical and whistler scattering rates. Here, $\zeta=v_{\|} / v$ is the cosine of the pitch angle. 
The calculation that follows is not without assumptions. Specifically we assume that once whistlers begin to grow they are able to scatter electrons through the full range of pitch angle. For oblique whistlers this is reasonable because of the multiple anomalous resonances and if the spectrum of waves is sufficiently broad. The assumption that the rate of scattering is independent on the pitch angle is based on the idea that electron pileup at a specific pitch angle would produce instabilities that resonate with electrons at that pitch angle and therefore facilitate scattering. Thus, the assumption is not that the whistler amplitudes are large, which is not expected in a system with very large ambient gradient scale lengths, but that resonant overlap in the quasilinear sense is a consequence of the broad wave spectrum - there are enough waves so that all electrons can undergo resonant interactions. We emphasize also that in the solar wind, where gradients scales are also large, observations reveal that the strahl electrons are scattered over a broad range of pitch angles (Cattell et al. 2021b). Finally, in the present calculation we assume that heat flux driven whistlers dominate electron scattering. Other scattering mechanisms associated with, for example, pressure anisotropy driven mirror modes have also been proposed (Komarov et al. 2016) but are not included in the present calculation.

The ambient ion velocity $U_{i}$ is included in the equations for completeness with the ordering $\epsilon \sim U_{i} / v_{t e}$. Transport is described by coupled equations for the electron density $n(x, t)$, the electron pressure $P_{e}(x, t)$, and the energy density of propagating whistler waves such that the total energy is conserved. The calculation parallels that carried out by Braginskii for the case of classical collisions (Braginskii 1965; Hassam 1980) with functional characteristics that parallel the equations describing cosmic ray transport limited by Alfvén wave scattering (Kulsrud \& Pearce 1969; Zweibel 2013; Thomas \& Pfrommer 2019).

A surprise is that the final equations describe both the drive of the whistlers by the release of energy associated with particle scattering by the waves and the saturation of the instability when the scattering rate exceeds a threshold. The onset of whistler growth is controlled by classical collisions and requires $L_{c}>L / \beta_{e}$ with $L_{c}=v_{t e} / \nu_{e i}\left(v_{t e}\right)$ the classical mean-free-path. The saturation of the instability takes place when $L_{w} \sim L / \beta_{e}$ with $L_{w} \sim v_{t e} / \nu_{w}\left(v_{t e}\right)$ the whistler scattering mean free path. Thus, the scattering mean free path of electrons in a high $\beta_{e}$ medium is always much smaller than 
the ambient scale length of the electron temperature even in the nominally collisionless domain. A second surprise is that the electron heat flux in the regime in which whistler scattering dominates classical collisions is not simply given by advection at the whistler phase speed $V_{w}$ but is controlled by a combination of advection and diffusion, which are of the same order. This follows from the diffusive heat flux, which is of order

$$
n \frac{v_{t e}^{2}}{\nu_{w}\left(v_{t e}\right)} \frac{T_{e}}{L} \sim n T_{e} v_{t e} \frac{L_{w}}{L} \sim n T_{e} \frac{v_{t e}}{\beta_{e}} \sim n T_{e} V_{w} .
$$

That the electron heat flux has contributions from both advection and diffusion was overlooked in earlier papers describing the results of simulations (Roberg-Clark et al. 2018a; Komarov et al. 2018), but is consistent with the analogous equations for the transport of cosmic rays (Thomas \& Pfrommer 2019).

The manuscript is organized as follows: in Sec. 2 we present an overview of the transport equations, including a description of their basic properties; in Sec. 3 we present the derivation of the transport equations with a discussion of the basic assumptions of the model; in Sec. 4 we present the results from computations of the propagation of an electron heat pulse that contrasts the results of transport in a classical collisional dominated regime with one with whistler scattering; and in Sec. 5 we discuss the implications of our results for the ICM. Finally in the Appendix we present a set of equations that combines the new description of electron thermal transport with the conventional magnetohydrodynamic (MHD) equations. The resulting Whistler-regulated MHD equations (W-MHD) are suitable for describing the full dynamics of ICM plasma over the full range of classical collisionality with none of the conventional constraints on mean free path.

\section{OVERVIEW OF THE ELECTRON TRANSPORT EQUATIONS}

Here we present an overview of the equations describing electron transport. The scattering operator representing classical collisions is energy dependent but only scatters the electrons in pitch angle and therefore only accurately models electron-ion collisions. The whistler scattering operator is also energy-dependent and scatters in pitch angle in a frame moving with a single velocity $V_{w} \ll v_{t e}$. The energy dependence of the whistler scattering rate is essential to correctly describe a finite heat flux 
while at the same time maintaining zero net current. Below we write three equations that describe the plasma density, the electron energy, and the energy associated with electron heat-flux driven whistler waves. The continuity equation is given by

$$
\frac{\partial}{\partial t} n+\nabla_{\|} n U=0
$$

with $U$ the ion drift speed along the ambient large-scale magnetic field (not including the whistler magnetic fluctuations). Because of the zero net current condition $U$ is also the mean electron drift speed. The equation for the electron energy is

$$
\frac{\partial}{\partial t}\left(\frac{3}{2} n T\right)+\nabla_{\|} Q-U \nabla_{\|} n T=V_{w}\left(\alpha_{10} \frac{\nu_{w e}}{\nu_{e}} n \nabla_{\|} T+F_{w}\right)
$$

where the parallel heat flux $Q$ is

$$
Q=\left(\frac{5}{2} U+\alpha_{10} \frac{\nu_{w e}}{\nu_{e}} V_{w}\right) n T-\kappa_{e} \nabla_{\|} T
$$

It includes the traditional advection of the enthalpy, $5 n T / 2$, with the fluid velocity $U$ and contributions from advection by the whistler waves (proportional to $V_{w}$ ) as well as from thermal conduction with conductivity $\kappa_{e}$,

$$
\kappa_{e}=\alpha_{12} \frac{n T}{m_{e} \nu_{e}} .
$$

In Eqs. (4)-(6) the $\alpha_{i}$ parameters labeled with subscripts are dimensionless and of order unity. They arise from averages of combinations of the scattering rates and powers of the particle velocity $v$ over Maxwellian distributions. Explicit expressions are given in Table 1. The total scattering rate $\nu_{e}=\nu_{e i}^{e}+\nu_{w e}$ is evaluated at the electron thermal speed $v_{t e}$ and

$$
\nu_{e i}^{e}=\frac{4 \pi e^{4} n \Lambda}{m_{e}^{2} v_{t e}^{3}} \quad \text { and } \quad \nu_{w e}=0.1 \Omega_{e} \frac{\varepsilon_{w}}{\varepsilon_{B}} .
$$

The whistler scattering rate $\nu_{w e}$ is given by the quasilinear form with $\varepsilon_{w}$ and $\varepsilon_{B}$ the energy densities of the whistler waves and the large scale magnetic field $B$, respectively (Lee 1982; Schlickeiser 1989), and $\Lambda$ is the Coulomb logarithm. We note that because we have discarded electron-electron the parallel conduction in Eq. (6) does not reduce to the Spitzer value (Hassam 1980). As discussed in 
the Appendix, the parameter $\alpha_{12}$ can be corrected to produce the Spitzer value. In this overview of the transport equations we assume that the temperature gradient is everywhere negative so that only whistlers traveling in the positive direction along $\mathbf{B}_{0}$ are unstable. The general equations presented in the Appendix include waves propagating in both directions along $\mathbf{B}_{0}$. In the absence of scattering by whistlers the equation for $n T$ reduces to the one-dimensional (1D) Braginskii equation (Braginskii 1965).

The advection of electron energy associated with the whistler wave propagation has been documented in PIC simulations (Roberg-Clark et al. 2018a; Komarov et al. 2018). However, the whistlerdriven advective heat flux becomes small when classical collisions dominate those associated with whistlers. This behavior was not explored in PIC simulations since classical collisions were not included. The thermal conduction term takes the classical form, being inversely proportional to the collision rate $\nu_{e}$. The collision rate includes both classical and whistler-driven scattering so that, even in the absence of classical collisions, whistler waves also produce diffusive transport. This result is consistent with the measured parallel diffusion of electrons scattered by heat-flux-driven whistlers in simulations (Roberg-Clark et al. 2018a; Komarov et al. 2018). However, the diffusive contributions to heat flux associated with whistler scattering were missed in previous discussions of these simulations. A wave-driven flux with a form similar to that of Eq. (5) also appears in equations describing the transport of cosmic rays (Kulsrud \& Pearce 1969; Zweibel 2013; Thomas \& Pfrommer 2019).

The two terms on the right side of Eq. (4), which are proportional to $V_{w}$, describe the extraction of electron thermal energy by whistler waves and the heating of electrons associated with whistler scattering. The energy extraction term is proportional to the local temperature gradient and is negative for waves propagating down the gradient, corresponding to energy extraction from the gradient by whistlers. The dimensionless coefficient $\alpha_{10}$ leading the extraction term is only nonzero when the whistler scattering rate $\nu_{w}$ depends on the electron energy. For a scattering rate that has the powerlaw dependence, $\nu_{w} \propto v^{\gamma}$ with $\gamma>0, \alpha_{10}$ is proportional to $\gamma$ in the limit of both strong and weak classical scattering. The powerlaw form for the energy dependence of $\nu_{w}$ results from a quasilinear model in which electrons with higher energies resonate with longer wavelength modes (see 
Eq. 1) and longer wavelength modes have larger amplitudes in a system undergoing an energy cascade (Lee 1982; Schlickeiser 1989; Schlickeiser \& Miller 1998). Further discussion of the appropriate value for $\gamma$ is presented in Sec. 3. The term proportional to $F_{w}$ in Eq. (4) arises from the drag force between the whistlers and electrons and is given by

$$
F_{w}=\alpha_{11} m_{e} n \nu_{w e} V_{w}
$$

It is positive and drives electron heating. It is a consequence of the linear resonant damping of whistlers, which heats the electrons. This is discussed further in the context of the equation for the growth of whistlers by the ambient heat flux below. The balance between energy extraction from the temperature gradient and from the damping of whistlers by electrons that describes both the onset of whistler wave growth in a medium with classical collisions and the saturation of the whistler wave energy.

An evolution equation for the whistler wave energy $\varepsilon_{w}$ is required to close Eq. (4). The equation for $\varepsilon_{w}$ includes advection both by the plasma fluid with velocity $U$ and by the whistlers with velocity $V_{w} \sim v_{t e} / \beta_{e}$ as well as drive and loss contributions associated with the extraction and heating terms on the right side of Eq. (4). Balancing the energy gain of the whistlers with the energy loss from electrons yields the evolution equation

$$
\frac{\partial}{\partial t} \varepsilon_{w}+2 \nabla_{\|}\left(V_{w}+U\right) \varepsilon_{w}-U \nabla_{\|} \varepsilon_{w}=-V_{w}\left(\alpha_{10} \frac{\nu_{w e}}{\nu_{e}} n \nabla_{\|} T+F_{w}\right),
$$

where the second term on the left side of the equation is the Poynting flux and the third term is associated with the whistler pressure acting on ions. There is a corresponding term in the ion kinetic energy equation (see Eq. (A29)) so that the ions and whistlers can exchange energy through this pressure. Thus, the sum of Eqs. (4) and (9) plus the equation for ion kinetic energy yields an equation for overall energy conservation.

An important scientific goal is to establish the conditions under which whistler waves in a high $\beta_{e}$ system begin to grow even when there are ambient classical collisions. The onset condition for whistler amplification can be obtained from Eq. (9) by taking the limit in which the whistler wave 
energy $\varepsilon_{w}$ is small so that the whistler scattering rate $\nu_{w e}$ in Eq. (7) is much smaller than $\nu_{e i}$. The resulting equation for the rate of growth $\gamma_{w}$ of the wave energy is given by

$$
\gamma_{w}=-n V_{w} \frac{\partial \nu_{w e}}{\partial \varepsilon_{w}}\left(\alpha_{10} \frac{\nabla_{\|} T}{\nu_{e i}^{e}}+\alpha_{11} m_{e} V_{w}\right) .
$$

The drive term proportional to $\nabla_{\|} T$ extracts energy from electrons (see Eq. (4)) to destabilize the whistlers. The damping term can be evaluated explicitly using Eq. (7) to evaluate $\nu_{w e}$. The result for the whistler damping $\gamma_{w d}$

$$
\frac{\gamma_{w d}}{\omega_{0}} \sim 0.1 \frac{V_{w}}{v_{t e}} \beta_{e}
$$

where $\omega_{0}=\Omega_{e} / \beta_{e}$ is the characteristic whistler wave frequency. This scaling for whistler wave damping is identical to that obtained from the resonant interaction of electrons with oblique whistlers from kinetic theory. The onset condition for whistler growth is insensitive to the details of the scattering rate and is given by

$$
-\nabla_{\|} T>\frac{\alpha_{11}}{\alpha_{10}} m_{e} \nu_{e i}^{e} V_{w}
$$

or $\beta_{e} L_{c} / L>1$. A similar result came from a quasilinear analysis of whistler stability (Pistinner \& Eichler 1998). Above threshold the growth rate $\gamma_{w}$ of the whistler wave energy scales as

$$
\gamma_{w} \sim 0.1 \Omega_{e} \frac{L_{c}}{L} .
$$

A measure of the strength of whistler growth is given by a comparison with the rate at which whistlers transit down the temperature gradient $V_{w} / L$. The whistler growth rate exceeds the transit rate for $\beta>10 \rho_{e} / L$, which is easily satisfied since $\rho_{e}$ is many orders of magnitude smaller than the macroscale $L$. The implication is that once the threshold for whistler growth is exceeded, the whistlers will rapidly reach finite amplitude.

The saturation of the whistler waves is controlled by the balance between the drive and damping terms on the right side of Eq. (9). The drive term becomes independent of $\nu_{w e}$ when $\nu_{w e}>\nu_{e i}$ while the dissipation term, which is proportional to $F_{w}$ and $\nu_{w e}$ continues to increase as whistler growth continues. Thus, whistler growth continues until the drive and dissipation terms on the right side of 
Eq. (9) balance. This balance gives the rate of whistler scattering at saturation,

$$
\nu_{w e}=-\frac{\alpha_{10}}{\alpha_{11}} \frac{1}{m_{e} V_{w}} \nabla_{\|} T
$$

which yields the scaling for the whistler scattering rate $\nu_{w e} \sim \beta_{e} v_{t e} / L$ and the whistler scattering mean-free-path $L_{w}=v_{t e} / \nu_{w e} \sim L / \beta_{e}$. Thus, even when classical electron-ion collisions are weak, whistler driven scattering reduces the electron mean-free-path to a scale length that is small compared with the ambient temperature scale length $L$. Further, the saturated value of $\nu_{w e}$ is insensitive to the details of the whistler growth rate in Eq. (10). At saturation the whistler wave energy density $\varepsilon_{w}$ can be calculated by equating the expressions for $\nu_{w e}$ in Eqs. (7) and (14), which yields $\varepsilon_{w} \sim 10\left(\rho_{e} / L\right) n T \ll n T$. Thus, the whistler energy density remains small compared with the electron thermal energy density (Komarov et al. 2018).

The fast growth rate of whistlers compared with the transit time of the waves across the system suggests that the whistlers may reach an equilibrium even when the wave drive only weakly exceeds the threshold for instability. This state can be explored by discarding the time derivative in Eq. (9) so that the balance between the drive and dissipation terms on the right side of the equation must scale as $\nabla_{\|} V_{w} \varepsilon_{w} \ll V_{w} n T / L$. Therefore, in steady state the drive and dissipation terms on the right side of Eq. (4) must balance and their difference must be negligible compared with the contribution from the heat flux since $\nabla_{\|} Q \sim V_{w} n T / L$. The heat flux $Q$ therefore dominates the dynamics of the electron energy equation and $\nu_{e}$ is given by the balance of the drive and dissipation terms,

$$
\nu_{e}=\nu_{e i}^{e}+\nu_{w e}=-\frac{\alpha_{10}}{\alpha_{11}} \frac{1}{m_{e} V_{w}} \nabla_{\|} T
$$

This expression for $\nu_{e}$ is valid as long as the onset condition for whistler growth in Eq. (12) is satisfied so that $\nu_{w e}$ is positive. This expression for $\nu_{e}$ can then be inserted into the expression for the heat flux in Eq. (5), which yields

$$
Q=\left[\frac{5}{2} U+\left(\alpha_{10}\left(1-\frac{\nu_{e i}^{e}}{\nu_{e}}\right)+\frac{\alpha_{11} \alpha_{12}}{\alpha_{10}}\right) V_{w}\right] n T .
$$

This expression is valid above the threshold for whistler growth and reveals that, in the regime dominated by whistlers, the proportionality of the scattering rate to $\nabla_{\|} T$ causes the expected diffusive 
driven energy flux to become advective. At marginal stability where $\nu_{e}=\nu_{e i}$ the first term in the heat flux in Eq. (16) is zero and the second term in the heat flux reduces to the classical diffusive form. It remains to be seen whether the form of the heat flux given in Eq. (16) is useful in a system where the whistler transitions from a stable to unstable domain, which will happen in any system where the electron temperature peaks so that $\nabla_{\|} T$ is zero in a local region and there is no whistler drive.

\section{DERIVATION OF THE ELECTRON TRANSPORT EQUATIONS}

Having presented an overview of the electron transport equations, we proceed to outline the assumptions, ordering and derivation. The focus here is on the electron distribution function and resulting moments and the wave equation for the whistler waves. Because the ion momentum and energy equations are largely unchanged from the traditional MHD description (barring a small force term associated with the whistler pressure acting on the ions) the coupling to the ion momentum and energy equations are deferred to Appendix A where the complete set of MHD equations with coupled whistler dynamics is presented.

\subsection{Chapman-Enskog solution of the electron kinetic equation}

We begin with an equation for the distribution function $f\left(x, v_{\|}, v_{\perp}, t\right)$, where $x$ is the space variable parallel to the ambient magnetic field $\mathbf{B}_{0}$ and $v_{\|}$and $v_{\perp}$ are the velocities parallel and perpendicular to $\mathbf{B}_{0}$. Since the whistler waves in a high $\beta_{e}$ system have $k \rho_{e} \sim 1$ so that the whistler wave frequency is well below the electron cyclotron frequency (see Eq. 1), the electrons remain gyrotropic. The kinetic equation for electrons becomes

$$
\frac{\partial}{\partial t} f+v_{\|} \nabla_{\|} f-\frac{e}{m_{e}} E_{\|} \frac{\partial}{\partial v_{\|}} f=C(f)
$$

where a parallel electric field $E_{\|}$has been included to maintain zero net current. The zero current condition is valid even in a system with complex three-dimensional magnetic fields as long as the kinetic scales are ordered out of the dynamics (Drake et al. 2019; Arnold et al. 2019). The collision operator includes scattering by both classical electron-ion collisions and whistler waves

$$
C(f)=C_{e i}(f)+C_{w}(f)
$$


with

$$
C_{e i}=\frac{1}{2} \nu_{e i}(|\mathbf{v}-\mathbf{U}|) \frac{\partial}{\partial \mathbf{v}} \cdot\left(\overrightarrow{\vec{I}}|\mathbf{v}-\mathbf{U}|^{2}-(\mathbf{v}-\mathbf{U})(\mathbf{v}-\mathbf{U})\right) \cdot \frac{\partial}{\partial \mathbf{v}}
$$

and

$$
C_{w}=\frac{1}{2} \nu_{w}\left(\left|\mathbf{v}-\mathbf{U}-\mathbf{V}_{w}\right|\right) \frac{\partial}{\partial \mathbf{v}} \cdot\left(\overrightarrow{\vec{I}}\left|\mathbf{v}-\mathbf{U}-\mathbf{V}_{w}\right|^{2}-\left(\mathbf{v}-\mathbf{U}-\mathbf{V}_{w}\right)\left(\mathbf{v}-\mathbf{U}-\mathbf{V}_{w}\right)\right) \cdot \frac{\partial}{\partial \mathbf{v}}
$$

describing the respective types of scattering. To simplify the derivation, we include here only whistlers propagating in the positive direction, which is valid for a locally negative temperature gradient. The generalization to counter-propagating whistlers is straightforward and the results are presented in the Appendix. The collision operators in Eqs. (19) and (20) describe scattering only in pitch angle - in the frame moving with the net parallel plasma drift $U$ in the case of $C_{e i}$ and with respect to the whistler wave frame $U+V_{w}$ in the case of whistler waves. Both scattering rates have energy dependencies with $\nu_{e i} \propto v^{-3}$ and $\nu_{w} \propto v^{\gamma}$. It will follow from the solutions to Eq. (17) that the energy dependence of $\nu_{w}$ is crucial for maintaining a net whistler driven advective heat flux while at the same time maintaining zero net current. An important assumption in the model for whistler scattering is that the scattering preserves energy in the whistler wave frame, which follows from the fact that, for long wavelength oscillations in that frame, the electric field vanishes. An additional assumption, however, is that $\nu_{w}$ is independent of pitch angle. Such a model is supported by PIC simulations of heat-flux-driven whistlers in which the particles were isotropized in pitch angle. On the other hand, the amplitudes of whistlers in a real system are likely to saturate at much lower values than in the simulations because the temperature gradient scale lengths in real systems are far larger. As discussed in Sec. 2, the saturation amplitude of the whistler fluctuations $\varepsilon_{w}$ scales as $n T \rho_{e} / L \ll n T$. Quasilinear models of electron scattering based on an assumed spectrum of waves yield scattering rates that depend on the pitch angle through $\zeta=v_{\|} / v$. However, we argue that the spectrum of heat-flux-driven waves will evolve so that the scattering rate is insensitive to $\zeta$ since any pileup of the distribution function with pitch angle would lead to local growth of whistlers which would enhance the local scattering. For this reason, the whistler scattering rate is taken to be independent of pitch angle. 
Equation (17) is solved to second order in the parameter $\epsilon$ discussed at the end of Sec. 1. Fundamental to the ordering is the assumption that the collisional mean free path is short compared with the parallel system scale length $L$ and that both the mean drift speed $U$ and the whistler phase speed $V_{w}$ are small compared with the thermal speed so that the collision operators as well as $f$ can be expanded in powers of $\epsilon \sim V_{w} / v_{t e} \sim U / v_{t e}$. Thus, we begin by writing Eq. (17) to lowest order,

$$
0=C_{0}\left(f_{0}\right)
$$

with

$$
C_{0}=\frac{1}{2}\left(\nu_{e i}+\nu_{w}\right) \frac{\partial}{\partial \mathbf{v}} \cdot\left(\overrightarrow{\vec{I} v^{2}}-\mathbf{v v}\right) \cdot \frac{\partial}{\partial \mathbf{v}},
$$

whose solution is a Maxwellian with zero drift,

$$
f_{0}=n_{0}\left(\frac{m_{e}}{2 \pi T_{0}}\right)^{3 / 2} e^{-m_{e} v^{2} / 2 T_{0}} .
$$

Technically, $C_{0}$ only scatters in pitch angle so $f_{0}$ could be written as any function of $v^{2}$. However, we take $f_{0}$ to be a Maxwellian. To first order, the derivatives on the left side of Eq. (17) need to be included,

$$
\frac{\partial}{\partial t} f_{0}+v_{\|} \nabla_{\|} f_{0}-\frac{e}{m_{e}} E_{\|} \frac{\partial}{\partial v_{\|}} f_{0}=C_{0}\left(f_{1}\right)+C_{1}\left(f_{0}\right)
$$

where

$$
\begin{aligned}
C_{1}= & \frac{1}{2} \nu_{w}(v) \frac{\partial}{\partial \mathbf{v}} \cdot\left(-2 \overrightarrow{\vec{I} \mathbf{v}} \cdot\left(\mathbf{U}+\mathbf{V}_{w}\right)+\mathbf{v}\left(\mathbf{U}+\mathbf{V}_{w}\right)+\left(\mathbf{U}+\mathbf{V}_{w}\right) \mathbf{v}\right) \cdot \frac{\partial}{\partial \mathbf{v}} \\
& -\frac{\partial \nu_{w}}{\partial v^{2}} \mathbf{v} \cdot\left(\mathbf{U}+\mathbf{V}_{w}\right) \frac{\partial}{\partial \mathbf{v}} \cdot\left(\overrightarrow{\vec{I} v^{2}}-\mathbf{v v}\right) \cdot \frac{\partial}{\partial \mathbf{v}} \\
& +\left(\nu_{w} \rightarrow \nu_{e i}, \mathbf{V}_{w} \rightarrow 0\right)
\end{aligned}
$$

Both $C_{0}$ and $C_{1}$ preserve the number density and the kinetic energy when averaged over velocity and the mean drift of $f_{0}$ is zero so

$$
\frac{\partial n_{0}}{\partial t}=0, \quad \frac{3}{2} \frac{\partial P_{0}}{\partial t}=0
$$

with $P_{0}=n_{0} T_{0}$. Thus, the time derivative of $f_{0}$ in Eq. (24) can be neglected. The various derivatives acting on $f_{0}$ (from $\nabla_{\|}, \partial / \partial \mathbf{v}$ and $C_{0}$ ) can be readily evaluated. The inversion of $C_{0}\left(f_{1}\right)$ is simplified by noting that $f_{1}=f_{1}(x, v, \zeta, t)$ so that

$$
C_{0}\left(f_{1}\right)=\frac{\nu}{2} \frac{\partial}{\partial \zeta}\left(1-\zeta^{2}\right) \frac{\partial}{\partial \zeta} f_{1}
$$


which is then readily inverted to yield the solution for $f_{1}$,

$$
f_{1}=-\frac{1}{\nu} \frac{v \zeta f_{0}}{T_{0}}\left[\frac{1}{n_{0}} \nabla_{\|} P_{0}+\left(\frac{m_{e} v^{2}}{2 T_{0}}-\frac{5}{2}\right) \nabla_{\|} T_{0}+e E_{\|}-m_{e}\left(\nu_{w}\left(V_{w}+U\right)+\nu_{e i} U\right)\right] \text {. }
$$

The first order electron drift velocity $U_{1}$ can then be evaluated by multiplying $f_{1}$ by $v_{\|}=v \zeta$ and integrating over velocity. The result is Ohm's law,

$$
0=m_{e} \nu_{e}\left(U_{1}-U\right)=-\alpha_{1}\left(\frac{1}{n_{0}} \nabla_{\|} P_{0}+e E_{\|}\right)-\alpha_{2} \nabla_{\|} T_{0}+\alpha_{3} m_{e} \nu_{w e} V_{w},
$$

where we have invoked the zero current condition to require $U_{1}=U$. This equation then determines $E_{\|}$. The dimensionless parameters $\alpha_{1}, \alpha_{2}$, and $\alpha_{3}$ are given in Table 1 . The collision rates in Eq. (29) are evaluated at the electron thermal speed, $\nu_{e}=\nu_{w e}+\nu_{e i}^{e}$ with $\nu_{w e}=\nu_{w}\left(v_{t e}\right)$ and $\nu_{e i}^{e}=\nu_{e i}\left(v_{t e}\right)$. The term proportional to $\nabla_{\|} T_{0}$ in Eq. (29) is the thermal force and is only nonzero when the velocity dependence of the scattering rate is included. The first order electron heat flux $Q_{1}$ can also be evaluated by multiplying $f_{1}$ by $m_{e} v^{2} v_{\|} / 2$ and averaging over velocity. After eliminating $E_{\|}$using Eq. (29), the result for $Q_{1}$ is given by

$$
Q_{1}=\left(\frac{5}{2} U+\alpha_{10} \frac{\nu_{w e}}{\nu_{e}} V_{w}\right) n_{0} T_{0}-\kappa \nabla_{\|} T
$$

with $\kappa$ given in Eq. (6). When the whistler scattering rate is small, the heat flux reduces to the classical form, which includes advection as well as parallel thermal conduction.

To obtain the evolution equations for the electron density and temperature, it is necessary to write Eq. (17) to second order. It takes the form

$$
\frac{\partial}{\partial t} f_{1}+v_{\|} \nabla_{\|} f_{1}-\frac{e}{m_{e}} E_{\|} \frac{\partial}{\partial v_{\|}} f_{1}=C_{0}\left(f_{2}\right)+C_{1}\left(f_{1}\right)+C_{2}\left(f_{0}\right)
$$

where $C_{0}$ and $C_{1}$ are given in Eqs. (22) and (25). There are terms from the second order collision operator $C_{2}$ that arise from the velocity dependence of the collision rates $\nu_{e i}$ and $\nu_{w}$. However, these terms all cancel when computing the continuity and energy moments of Eq. (31) so the minimal required expression for $C_{2}$ is given by

$$
\begin{aligned}
C_{2}= & \frac{1}{2} \nu_{w}(v) \frac{\partial}{\partial \mathbf{v}} \cdot\left(\overrightarrow{\vec{I}}\left(U+V_{w}\right)^{2}+\left(\mathbf{U}+\mathbf{V}_{w}\right)\left(\mathbf{U}+\mathbf{V}_{w}\right)\right) \cdot \frac{\partial}{\partial \mathbf{v}} \\
& +\left(\nu_{w} \rightarrow \nu_{e i}, \mathbf{V}_{w} \rightarrow 0\right) .
\end{aligned}
$$


The evolution equation for the plasma density is obtained by integrating Eq. (31) over the velocity. All of the collision terms on the right side of the equation as well as the $E_{\|}$term integrate to zero, leaving the continuity equation as written in Eq. (3). Note that it is not necessary to evaluate $f_{2}$ to obtain the density evolution equation since $C_{0}\left(f_{2}\right)$ integrates to zero. The equation for the evolution of the electron energy is obtained by multiplying Eq. (31) by $m_{e} v^{2} / 2$ and integrating over the velocity. As in the continuity equation, $f_{2}$ drops out since in a stationary frame where $C_{0}$ is evaluated the scattering does not change the particle energy. The electron energy evolution equation takes the form presented in Eq. (4).

As discussed in Sec. 3, the temperature gradient drive of the whistlers is only nonzero if the whistler scattering rate is energy dependent. The energy dependence of the whistler-driven scattering rate was not evaluated in PIC simulations of heat flux driven whistlers (Roberg-Clark et al. 2016, 2018a; Komarov et al. 2018) but the bounce frequency of electrons trapped in large-amplitude whistlers as seen in the simulations increases with the particle energy (Karimabadi et al. 1990). However, in real systems in which the temperature gradient scale length is large, the whistler wave amplitude will be small (see discussion in Sec. 3) and with a broader spectrum of waves than documented in the PIC simulations. The quasilinear scattering rate for an assumed spectrum of low-frequency Alfvén waves has been calculated previously (Lee 1982; Schlickeiser 1989; Schlickeiser \& Miller 1998). The velocity dependence of the scattering rate takes the form of a powerlaw $v^{\gamma}$ where $\gamma=q-1$ and where the wave energy spectrum was assumed to have a powerlaw form $k^{-q}$. Since the whistler waves of interest in electron scattering are also sub-cyclotron these earlier quasilinear results also apply to the electrons' response to whistlers. The spectrum of whistler waves in PIC simulations of heat flux driven whistlers fell off steeply (Roberg-Clark et al. 2018a) but the limited spectral range of the simulations likely impacted the results. The cascade of electron-MHD turbulence has also been explored (Biskamp et al. 1999) and yielded powerlaw spectra that scaled like $k^{-7 / 3}$ so that $\gamma \sim 4 / 3$. Thus, we take the whistler scattering rate to take the quasilinear form with a powerlaw dependence on velocity:

$$
\nu_{w}=0.1 \Omega_{e} \frac{\varepsilon_{w}}{\varepsilon_{B}}\left(v / v_{t e}\right)^{\gamma},
$$


with $\gamma \sim 4 / 3$. The numerical factor of 0.1 in Eq. (33) is based on the results of PIC simulations (Roberg-Clark et al. 2018a) rather than a detailed quasilinear calculation. However, as shown in Sec. 2, while this factor impacts the rate of growth of whistlers, it does not control their saturation and the associated saturated value of $\nu_{w e}$. Since the time scale for whistler growth is short compared with dynamical timescales, which vary as $L / V_{w}$, the details of the whistler growth rate are not important. More important is the onset criterion for whistler growth, which, as shown in Sec. 2, is independent of the factors appearing in Eq. (33).

\subsection{Whistler wave equation}

Finally, we present a derivation of Eq. (9), which describes the whistler energy transport. The extraction and heating terms follow from the transfer of energy between the electrons and whistlers through the scattering process. The calculation that leads to the advection terms on the left side of the equation, which are important in describing the propagation of the whistlers and evaluating their interaction with the bulk fluid, is presented here. However, to simplify the equations we limit the calculation to a simple system with straight magnetic field lines. The more general case is presented in the Appendix. We start with Faraday's law for the perturbed whistler magnetic field,

$$
\frac{\partial}{\partial t} \delta \mathbf{B}_{w}+c \nabla \times \delta \mathbf{E}_{w}=0,
$$

where $\delta \mathbf{B}_{w}$ and $\delta \mathbf{E}_{w}$ are the transverse magnetic and electric field of the whistler wave. Although the scattering of electrons by the whistlers requires that the waves be oblique with respect to the ambient magnetic field, here we explore only the transport along the ambient magnetic field and therefore consider a simple 1D model of the wave dynamics. Since the full 3D MHD equations will be displayed in the Appendix, the convection velocity $\mathbf{U}$ of the MHD fluid is taken as a general vector. Taking the dot product of Eq. (34) with $\delta \mathbf{B}_{w}$ and completing some vector algebra, we obtain an equation for the wave energy $\varepsilon_{w}=\left|\delta \mathbf{B}_{w}\right|^{2} / 8 \pi$

$$
\frac{\partial}{\partial t} \varepsilon_{w}+\frac{c}{4 \pi} \nabla \cdot\left(\delta \mathbf{E}_{w} \times \delta \mathbf{B}_{w}\right)+\delta \mathbf{J}_{w} \cdot \delta \mathbf{E}_{w}=-V_{w}\left(\alpha_{10} \frac{\nu_{w e}}{\nu_{e}} n \nabla_{\|} T+F_{w}\right),
$$

where we have used Ampère's law without the displacement current and have included the scattering terms on the right-hand side. To evaluate $\delta \mathbf{J}_{w} \cdot \delta \mathbf{E}_{w}$, we first note that $\delta \mathbf{J}_{w}=-n e \delta \mathbf{v}_{w}$ and 
An equation for the whistler electric field $\mathbf{E}_{w}$ is obtained from the linearized electron equation of motion with no inertia

$$
0=\delta \mathbf{E}_{w}+\frac{1}{c} \delta \mathbf{v}_{w} \times \mathbf{B}+\frac{1}{c} \mathbf{U} \times \delta \mathbf{B}_{w}
$$

where the electron streaming velocity $\mathbf{U}$ is equal to that of the ions because of the current in an MHD systems is zero unless Hall terms in Ohm's law are retained (Drake et al. 2019; Arnold et al. 2019). In the Poynting flux the whistler electric field $\delta \mathbf{E}_{w}$ takes the form,

$$
\delta \mathbf{E}_{w}=-\frac{1}{c}\left(\mathbf{U}+\mathbf{V}_{w}\right) \times \delta \mathbf{B}_{w}
$$

This is equivalent to transforming from the whistler wave frame, where the electric field is zero, into the laboratory frame. The Poynting flux takes the form

$$
\mathbf{S}_{w}=\frac{c}{4 \pi} \delta \mathbf{E} \times \delta \mathbf{B}=\left(2\left(U_{\|}+V_{w}\right) \mathbf{b}+\mathbf{U}_{\perp}\right) \varepsilon_{w}
$$

Using Eq. (36) to calculate $\delta \mathbf{E}_{W}$, the heating term can be written as $\delta \mathbf{J} \cdot \delta \mathbf{E}_{w}=\mathbf{U} \cdot \mathbf{F}_{p w}$ where $\mathbf{F}_{p w}$ is the whistler ponderomotive force acting on the electrons, which is given by

$$
\mathbf{F}_{p w}=-\frac{n e}{c} \delta \mathbf{v}_{w} \times \delta \mathbf{B}_{w}
$$

The right side is easily evaluated using Ampère's law for $\mathbf{v}_{w}$ and carrying out some vector algebra. The resulting expression for the ponderomotive force is

$$
\mathbf{F}_{p w}=-\mathbf{b b} \cdot \nabla \varepsilon_{w}
$$

Inserting $\mathbf{F}_{p w}$ and $\mathbf{S}_{w}$ into Eq. (35) yields the transport equation for $\varepsilon_{w}$ given in Eq. (9). The interaction between the whistler radiation pressure and the ions through the last term on the left side of the whistler transport equation requires a corresponding term in the ion momentum equation. This arises when the individual electron and ion momentum equations are added to produce the onefluid momentum equation. For completeness the full set of MHD equations along with the whistler constrained electron energy transport equations are presented in the Appendix. The results in the Appendix generalize the ponderomotive force to the case when magnetic fields have curvature. 


\section{SIMULATIONS OF ELECTRON THERMAL TRANSPORT WITH WHISTLER SCATTERING}

While the basic characteristics of whistler limited transport have been discussed in Sec. 2, here we show the results of numerical solutions of the coupled equations for the electron temperature $T_{e}$ and the whistler energy density $\varepsilon_{w}^{ \pm}$. For simplicity, we freeze the ions so that the bulk flow $U$ is zero and the plasma density remains constant. In the first test case we consider a system with an initial temperature profile and temperatures at the boundaries specified such that the temperature gradient is negative. We assume zero slope boundary conditions on so that the boundary temperatures can float. The whistler waves are assumed to have small amplitude at $t=0$ so that classical scattering dominates the early evolution. Because the temperature gradient is zero at the boundaries, the threshold for whistler growth in Eq. (12) is not satisfied close to the boundaries so the whistler wave amplitude remains small there. As a consequence, there is little heat flux through the boundaries and the integrated total energy (electron plus whistlers) in the system is conserved. Because the gradient of the temperature in the system is zero or negative everywhere, only rightward propagating waves are included since leftward propagating waves are damped.

It is convenient to normalize the equations to reduce the number of free parameters. The temperature is normalized to its value on the left boundary, $T_{0}$, and the whister wave energy to $n_{0} T_{0}$. Lengths are normalized to the length of the computational domain $L$ and time to the transit time of the whistler across the system $L / V_{w 0}$ with $V_{w 0}$ based on the parameters $T_{0}$ and $\beta_{0}$ of the left boundary. The scattering rate $\nu_{e}$ is normalized to the nominal saturated whistler scattering rate $\beta_{0} v_{t e 0} / L$ written below Eq. (14). The resulting normalized coupled equations for $T$ and $\varepsilon_{w}^{ \pm}$take the simple form

$$
\frac{3}{2} \frac{\partial}{\partial t} T+\nabla_{\|} Q=\left(\tilde{V}_{\mathrm{st}}^{+}-\tilde{V}_{\mathrm{st}}^{-}\right) \nabla_{\|} T+H_{w}^{+}+H_{w}^{-},
$$

where for notational simplicity we have not relabeled the variables (e.g., $\left.T / T_{0} \Rightarrow T\right)$. In Eq. (41)

$$
Q=\left(\tilde{V}_{\mathrm{st}}^{+}-\tilde{V}_{\mathrm{st}}^{-}\right) T-\kappa_{e} \nabla_{\|} T,
$$


with $\kappa_{e}=T / \nu_{e}, \nu_{e}=\nu_{e i}+\nu_{w e}^{+}+\nu_{w e}^{-}$and

$$
\nu_{e i}=\left(\frac{L}{\beta_{0} L_{c 0}}\right) \frac{1}{T^{3 / 2}}, \quad \nu_{w e}^{ \pm}=0.1 \varepsilon_{w}^{ \pm} \frac{L}{\rho_{e 0}} .
$$

The streaming velocities $\tilde{V}_{\text {st }}^{ \pm}$are defined as

$$
\tilde{V}_{\mathrm{st}}^{ \pm}=V_{w} \frac{\nu_{w e}^{ \pm}}{\nu_{e}}
$$

and $V_{w}=T^{-1 / 2}$. The heating functions take the form

$$
H_{w}^{ \pm}=V_{w}^{2}\left(\nu_{w e}^{ \pm}+\frac{\nu_{w e}^{+} \nu_{w e}^{-}}{\nu_{e}}\right)
$$

The equation for the whistler wave energy $\left(\varepsilon_{w e}^{ \pm} / n_{0} T_{0} \Rightarrow \varepsilon_{w}^{ \pm}\right)$is

$$
\frac{\partial}{\partial t} \varepsilon_{w}^{ \pm} \pm 2 \nabla_{\|} V_{w} \varepsilon_{w}^{ \pm}=\mp \tilde{V}_{\mathrm{st}}^{ \pm} \nabla_{\|} T-H_{w}^{ \pm} .
$$

The equations have two important free parameters, the collisionality parameter $L / \beta_{0} L_{c 0}$, which for values less than unity, leads to whistler growth and the Larmor radius parameter $L / \rho_{e 0}$ which controls the rate of growth of whistlers compared with the whistler transit time across the domain - a large value of $0.1 L / \rho_{e 0}$ indicates that in the unstable domain the whistlers will reach large amplitude in a time short compared with the global evolution of the system. For simplicity, we have set the various parameters $\alpha_{i}$ to unity since their values do not significantly impact the dynamics.

For computational reasons we do not actually evolve the whistler wave energy as written in Eq. (46). The equation leads to numerical problems in spatial locations where $\varepsilon_{w}^{ \pm} \Rightarrow 0$ because the time step can cause $\varepsilon_{w}$ to become negative. However, both of the terms on the right side of the equation are proportional to $\nu_{w e}^{ \pm}$and therefore to $\varepsilon_{w}^{ \pm}$. Thus, the equation can be divided by $\varepsilon_{w}^{ \pm}$and the equation can be written as an evolution equation for $\ln \varepsilon_{w}^{ \pm}$. This is in effect a stretching transformation for $\varepsilon_{w}^{ \pm}$around zero. The coupled Eqs. (41) and (46) are solved with the 1D Galerkin/Petrov-Galerkin method (Skeel \& Berzins 1990) using MATLAB.

In Fig. 1 we show cuts of (a) the temperature profile, (c) the whistler wave energy profile $\varepsilon_{w}^{+}$and associated whistler scattering rate $\nu_{w e}^{+}$and (d) the profile of the ratio of $\nu_{e}=\nu_{e i}^{e}+\nu_{w e}^{+}$to $\nu_{e i}^{e}$ at several times during the growth phase of the whistler waves $(t \in(0.0 .15))$. The leftward propagating whistler 
is stable for this simulation since $\nabla_{\|} T$ is never positive. The parameters for this simulation were $L / \beta_{0} L_{c 0}=0.05, L / \rho_{e}=1500$ with the initial temperature profile given by $1.5-0.5 \tanh (x-2.5)$. At the beginning of the evolution when $\nu_{w e}^{+}$is small, $\nu_{e}=\nu_{e i} \sim L / \beta_{0} L_{c 0}=0.05$ so $\kappa \sim 20$. The initial temperature evolution is rapid. However, the whistlers also grow rapidly until $\nu_{e} \sim 1$ and the evolution of the temperature slows dramatically. For comparison, the evolution of the temperature is shown in (b) with the whistler wave energy set to zero, corresponding to classical transport. The flattening of the temperature takes place much more rapidly in the absence of the suppression of transport by whistlers.

The time evolution of the temperature during the phase when whistler growth has saturated $(t \in$ $(0,1.5))$ is shown in Fig. 2(a). The plots in (a), (b) and (c) are as in Fig. 1. During this time the profiles of the whistler wave amplitude and scattering rate $\nu_{w e}$ evolve slowly, remaining in the saturated state defined by Eq. (14). That the temperature is advected by the whistlers can be seen by the trajectories of the location of the peak of the temperature gradient and of the whistler energy spectrum shown in (d). Both peaks propagate together because the whistlers rapidly reach equilibrium with the local temperature gradient as given in Eq. (14). The peaks propagates with nearly constant velocity of order unity, which corresponds to $V_{w}$ in our normalized system.

We now consider a more complex initial temperature profile with regions of positive and negative temperature gradient so that whistlers propagating to the left and right $\varepsilon_{w}^{ \pm}$must be included. The parameters for this simulation were the same as in Fig. 1 but with an initial temperature profile given by $2.0-\tanh (x-5 / 3)+0.5 \tanh ((x-10 / 3) / 0.5)$. As in the simulation of Fig. 1, we show in Fig. 3 cuts of (a) the temperature profile, (c) the whistler wave energy profiles $\varepsilon_{w}^{ \pm}$and associated whistler scattering rates $\nu_{w e}^{ \pm}$and (d) the profile of the ratio of $\nu_{e}=\nu_{e i}^{e}+\nu_{w e}^{+}+\nu_{w e}^{-}$to $\nu_{e i}^{e}$ at several times during the growth phase of the whistler waves $(t \in(0.0,0.1))$. As in the previous simulation, the temperature evolution is initially rapid. However, the whistlers grow rapidly with rightward propagating whistlers growing in the region of negative temperature gradient and the leftward propagating whistlers on the positive temperature gradient. Once $\nu_{e} \sim 1$ the evolution of the temperature slows dramatically. For comparison, the evolution of the temperature is shown in (b) with the whistler wave energy set 
to zero, corresponding to classical transport. Again the flattening of the temperature takes place much more rapidly in the absence of the suppression of transport by whistlers. An important result from the simulation with bidirectional whistlers is the absence of a region of significant overlap of the oppositely propagating waves. This is a consequence of the threshold for whistler growth given in Eq. (12). For a shallow temperature gradient classical collisions prevent whistler onset so there is always a stable band between the regions where the right and left going waves are unstable. The consequence is that two classes of waves develop in spatially distinct regions of space. This differs from the case of cosmic ray transport limited by Alfvén waves where counter-streaming waves can develop provided the wave damping rate is sufficiently low.

In Fig. 4 we show the late time evolution of the profile shown in Fig. 3. In (a) is the whistler limited profile evolution and in (b) the classical transport result. In the case of whistler limited transport the temperature minimum fills in as the temperature gradients on the left and right propagate towards the middle to fill in the temperature dip. This behavior is perhaps even more evident in (c), which displays the evolution of the whistler energy spectra. The peaks of the spectra move towards the temperature minimum, following the location of the maximum temperature gradient. $\varepsilon_{w}^{+}$moves to the right and $\varepsilon_{w}^{-}$moves to the left. In Fig. 5, the trajectory of the peak of the spectrum of $\varepsilon_{w}^{+}$is shown in (a) and that of $\varepsilon_{w}^{-}$is shown in (b). Again, the propagation velocities are of order unity and reflect the motion of the location of the maximum temperature gradient.

\section{DISCUSSION AND CONCLUSIONS}

A set of transport equations for electron energy that includes both classical electron-ion collisions and self-consistent scattering by whistler waves has been developed. The whistlers are driven unstable by the electron temperature gradient along the ambient magnetic field. For temperature scale lengths below the critical value $L=\beta_{e} L_{c}$, with $L_{c}$ the classical electron mean-free-path and $\beta_{e}$ the ratio of electron to magnetic pressure, whistler waves will rapidly grow and reduce electron transport below that based on the classical Spitzer conductivity. For typical values of $L_{c}$ in the ICM ranging from $0.1-1 \mathrm{kpc}$ and $\beta_{e} \sim 100$, large regions of the ICM are likely to be controlled by whistler-limited thermal transport. 
While the impact of whistler constrained thermal transport on the dynamics of the ICM will require detailed calculations beyond the scope of the present paper, simple scaling arguments suggest the broad importance of the results for the ICM. In the following, we will show that (1) the suppression of thermal conduction decreases the characteristic length below which thermal instability is suppressed (Field 1965), (2) the temperature dependence of heat flux transitions from $T_{e}^{7 / 2} / L$ to $V_{w} n T_{e} \sim T_{e}^{1 / 2}$ as a system transitions to the regime where classical collisions are weak, (3) the magneto-thermal (MTI) and heat-flux-driven buoyancy (HBI) instabilities are impaired or completely inhibited by whistler limited heat flux, and (4) the heat flux constraint allows sound waves, which are significant players in heating the cool cores of the ICM plasma, to propagate greater distances from the central black hole, consistent with observations. Finally, we discuss the potential of direct measurements in the high $\beta$ solar wind to validate the new model.

\subsection{The impact on thermal condensation}

Further constraints on electron thermal transport from whistler scattering will make it even less likely that heat conduction from the outer regions of the ICM to the cool core can limit the radiative collapse at the largest scales. On the other hand, an important question is whether the local dynamics of radiative instabilities that are likely to develop within the cool core will be altered compared with the classical model. In the case of an isobaric perturbation about an equilibrium in which radiative cooling is balanced by some local heat source, the suppression of thermal transport relative to the classical Spitzer value will decrease the Field length (Field 1965), thereby extending the domain of thermal instability down to smaller spatial scales. Further, the significant consequences of whistler limited transport can be more clearly identified by considering a state in which radiative cooling is balanced by a non-zero divergence of the heat flux. In the simplest one-dimensional model of an isobaric condensation, the heat flux in the classical model scales as $T^{7 / 2} / L$ with $L$ the ambient temperature scale length. To maintain a constant heat flux into the condensing region where the temperature is dropping requires the local temperature gradient to increase or $L$ to decrease. The reduction in $L$ pushes the system towards the whistler unstable domain. Specifically, the heat flux 
$Q$ scales as

$$
Q \sim \frac{L_{c}}{L} n T_{e} v_{t e}
$$

so the whistler instability criterion can be written as

$$
\frac{L}{\beta_{e} L_{c}} \sim \frac{n T_{e} v_{t e}}{\beta_{e} Q} \sim \frac{B^{2}}{8 \pi Q} v_{t e}<1
$$

Thus, in a 1D system where $B$ is a constant, the threshold for whistler onset will be crossed when

the temperature is low enough. At that point the scaling of the heat flux will change from $T^{7 / 2} / L$ to $V_{w} n T_{e} \sim T_{e}^{1 / 2}$. Thus, the dynamics of condensation is likely be substantially changed in light of the new transport model.

The temperature gradients across cold fronts can be quite large and, depending on the strength of the locally draped magnetic field, are likely to drive whistlers that in turn can control transport and the structure of these fronts. The equations developed here provide a fully self-consistent framework for exploring the dynamics and structure of cold fronts with no constraints on the possible breakdown of the classical model of thermal transport.

\subsection{The impact of heat flux suppression on anisotropic transport instabilities}

A pure hydrodynamic and gravitationally stratified plasma is unstable to perturbations that cause convective motions if the entropy is a decreasing function of radius, i.e., if $\partial \ln K / \partial \ln r<0$ (Schwarzschild 1906), where $K=P \rho^{-\Gamma}$ is the entropic function labeling an adiabatic curve, $\rho$ is the mass density, and $\Gamma=5 / 3$ is the ratio of specific heats. The entropy profiles of all observed galaxy clusters are increasing functions of radius, which should render them convectively stable (Cavagnolo et al. 2008). If a fluid element in a stably stratified atmosphere is adiabatically displaced in radius from its equilibrium position by $\delta r$, it experiences a buoyant restoring force per unit volume (Ruszkowski \& Oh 2010)

$$
F_{\text {adiab }}=\rho \ddot{r}=-\frac{\rho g}{\Gamma} \frac{\partial \ln K}{\partial r} \delta r
$$


where $g$ is the gravitational acceleration, that causes oscillations around its equilibrium position at the classical Brunt-Väisälä frequency $N$ where

$$
N^{2}=\frac{g}{\Gamma r} \frac{\partial \ln K}{\partial \ln r}
$$

More fundamentally, the Brunt-Väisälä frequency is the limiting frequency of linear internal gravity waves (which have frequency $\omega_{\mathrm{gw}}^{2}=k_{h}^{2} N^{2} /\left|k^{2}\right|$ where $k_{h}$ is the component of the wavenumber orthogonal to the gravity gradient), so real $N$ translates into linear stability of the atmosphere. However, if an external turbulent driving force is larger than the buoyant restoring force then it can overcome the stable equilibrium and induce mixing.

This paradigm experiences a fundamental change in the weakly collisional, magnetized plasma of a galaxy cluster because it changes the response of the plasma to perturbations. Provided there is a radial temperature gradient, $\partial T_{e} / \partial r \neq 0$, anisotropic thermal conduction along the mean magnetic field causes the ICM to be almost always buoyantly unstable regardless of the sign of the temperature and entropy gradients. If $\partial T_{e} / \partial r>0$ (which applies to the central cooling regions in cool core clusters) the ICM is unstable to the heat-flux-driven buoyancy instability (HBI, Quataert 2008) provided that there are regions where the magnetic field is mostly radially aligned. If $\partial T_{e} / \partial r<0$ (which applies to all clusters on large scales and is a consequence of hydrostatic rearrangement in the presence of the universal dark matter potential) the ICM is unstable to the magneto-thermal instability (MTI, Balbus 2000) provided that there are regions where the magnetic field is mostly horizontally aligned. Using a linear stability analysis (Kunz 2011) and non-linear simulations (Kunz et al. 2012) it was shown that anisotropic viscosity (i.e., Braginskii pressure anisotropy) effects the MTI and HBI growth rates by stifling the convergence/divergence of magnetic field lines, but cannot suppress it. When field geometries are choosen such as to stabilize the HBI/MTI, the system is still subject to related overstabilities (Balbus \& Reynolds 2010). In consequence, any perturbation will be convectively unstable and cause instant mixing of the thermal plasma because it lacks a restoring force that provides an energy penalty (Sharma et al. 2009) which facilitates advection and turbulent mixing of AGN injected energy (Kannan et al. 2017). In the saturated state of these anisotropic transport 
instabilities, the buoyant restoring force is altered to $\left|F_{\text {cond }}\right| \sim \rho g\left(\partial \ln T_{e} / \partial r\right) \delta r$, and replaces the restoring force of Eq. (49) that is based on the classic Schwarzschild criterion (Sharma et al. 2009).

Kinetic plasma physics and finite Larmor-radius effects can however significantly impact those HBI/MTI instabilities, which have been found using a magnetofluid (i.e. Braginskii) description. While at long wavelengths (the 'drift-kinetic' limit), a kinetic analysis reinforces the MTI and its Alfvénic counterpart, at sub-ion-Larmor scales, there is an overstability driven by the electrontemperature gradient of kinetic-Alfvén drift waves whose growth rate is even larger than the standard MTI (Xu \& Kunz 2016). While the effective heat conductivity can locally be supressed by the ion-scale mirror instability (Komarov et al. 2016; Riquelme et al. 2016), recent work found that the nonlinear saturation of the MTI is not significantly modified (Berlok et al. 2021). Given that the predicted reduction in parallel thermal transport can be significant in our picture of whistler-mediated thermal conduction, an important question is whether this reduction might affect these large-scale MTI and HBI fluid instabilities. Formally, the current treatments of MTI and HBI explicitly assume diffusive parallel heat transport and so would need to be reworked with our modified scheme that highlights the importance of advective transport. However, as a first approach, it is possible to argue physically. The MTI/HBI rely on three properties: (i) the presence of a radial temperature gradient, $\partial T_{e} / \partial r \neq 0$, (ii) the large ratio of electron thermal transport along to that across the ambient magnetic field and (iii) a short conduction time in comparison to the buoyancy and advection time scales on the length scales considered. ${ }^{1}$

The perpendicular thermal transport arising from the whistler instability has not been explored in detail. The invariance of the canonical momentum in the symmetry direction of a 2D PIC model constrains particle motion perpendicular to $\mathbf{B}$ so that the exploration of perpendicular transport requires the PIC modeling to be carried out in a more computationally challenging 3D system. On the other hand, based on the basic characteristics of the heat-flux-driven whistler, we can estimate

${ }^{1}$ As noted by our referee, there is an additional property: the viscous time cannot be too short in comparison to the buoyancy time. Because the conduction time and the viscous time are related (in Braginskii's MHD), one cannot take the limit of fast conduction while neglecting the anisotropic viscous stress (Kunz 2011), which also suppresses the HBI by stifling the convergence/divergence of magnetic-field lines that is responsible for generating the buoyant motions. 
the perpendicular transport. The characteristic transverse scale of the whistler turbulence is the electron Larmor radius $\rho_{e}$ so that a reasonable estimate of the cross-field diffusion is

$$
D_{\perp} \sim \rho_{e}^{2} \nu_{w e}
$$

The parallel transport is given by

$$
D_{\|} \sim \frac{v_{t e}^{2}}{\nu_{w e}} .
$$

Thus, the ratio $R_{\text {trans }}$ of the parallel to perpendicular transport is given by

$$
R_{\text {trans }}=\frac{D_{\|}}{D_{\perp}} \sim\left(\frac{L}{\beta \rho_{e}}\right)^{2} \sim 3 \times 10^{26}\left(\frac{L}{30 \mathrm{kpc}}\right)^{2}\left(\frac{T_{e}}{10^{7} \mathrm{~K}}\right)^{-1}\left(\frac{B}{1 \mu \mathrm{G}}\right)^{2},
$$

where we have used the saturated whistler scattering rate $\nu_{w e}=\beta_{e} v_{t e} / L$ given in Eq. (14). Thus, the anisotropy ratio remains extreme, in spite of the factor $\beta_{e}$ in the denominator of Eq. (53).

While whistler-wave scattering does not qualitatively change the anisotropic character of heat transport and hence the physical basis for the MTI and HBI, the scale $\ell$ that can go unstable is modified. Assuming a hydrostatic atmosphere, these instabilities only grow at interesting rates provided the characteristic time scale at which conduction acts on a given perturbation is much shorter than the buoyancy time scale, which we identify with the inverse Brunt-Väisälä frequency, $N$, and obtain the condition

$$
\tau_{\text {cond }}=\frac{\ell}{V_{w}}=\frac{\ell \beta_{e}}{v_{t e}} \ll N^{-1}=\left(\frac{g}{\Gamma r} \frac{\partial \ln K}{\partial \ln r}\right)^{-1 / 2} .
$$

This time scale ordering is the basis for the excitation of the MTI and HBI and ensures quasiisothermality along a given field line (Quataert 2008). Because tangled magnetic field lines have a significant portion of azimuthal components not aligned with the vertical buoyancy direction, this adds furthermore to the large separation of time scales. We assume an NFW dark matter density profile (Navarro et al. 1997) which is characterized by a scale radius, $r_{s}$, and a mass density at the scale radius, $\rho_{s}$, so that the gravitational acceleration in the inner regions at radii $r<r_{s}$ is given by

$$
g_{0}=\frac{G M(<r)}{r^{2}}=2 \pi G \rho_{s} r_{s}=\text { const. }
$$


where $G$ is Newton's constant. We furthermore assume an exponentially stratified atmosphere where the pressure scale height is given by $h=v_{t p}^{2} / g_{0}$ (where $v_{t p}$ is the isothermal sound speed) so that the critical length scale for instability is

$$
\ell \ll \frac{v_{t e}}{\beta_{e}} N^{-1}=\sqrt{\frac{\Gamma}{\Gamma-1}} \frac{v_{t e} v_{t p}}{\beta_{e} g_{0}} \sim 20\left(\frac{\sqrt{T_{e} T_{p}}}{10^{7} \mathrm{~K}}\right)\left(\frac{\beta_{e}}{100}\right)^{-1}\left(\frac{g_{0}}{10^{-8} \mathrm{~cm} \mathrm{~s}^{-2}}\right)^{-1} \mathrm{kpc} .
$$

On larger length scales, conduction is slower than buoyancy and the system transitions to obey the classical Schwarzschild criterion for convective instability, $\partial \ln K / \partial \ln r<0$. However, on scales smaller than $\ell$, there is no restoring force associated with the entropy gradient and passive scalars such as metals should get easily mixed with the surrounding ICM as explained above. This picture is predicated upon the formal applicability of the MTI and HBI but, for realistic magnetic field strengths in clusters with $\beta_{e} \sim 100$, magnetic tension provides an additional restoring force and can suppress a significant fraction of HBI-unstable modes, thus either impairing or completely inhibiting the HBI on scales smaller than $\sim 50-70 \mathrm{kpc}$, depending on the unknown magnetic field coherence length and the fraction of volume partitioned with these intermittent strong magnetic fields (Yang \& Reynolds 2016). These considerations strongly constrain the applicability of the MTI and HBI and call for a careful assessment as to whether they are excited in linear theory at all in the presence of whistlermediated thermal conduction.

If there are bulk flows in the ICM, the condition of Eq. (56) is modified and we require that the conductive time scale is much shorter than the advective time scale for the MTI and HBI to be excited, which can be rewritten into a condition for the advection velocity,

$$
U \ll V_{w}=\frac{v_{t e}}{\beta_{e}} \sim 100\left(\frac{T_{e}}{10^{7} \mathrm{~K}}\right)^{1 / 2}\left(\frac{\beta_{e}}{100}\right)^{-1} \mathrm{~km} \mathrm{~s}^{-1} .
$$

Both conditions of Eqs. (56) and (57) are constraining and may severely limit the applicability of the MTI and HBI in galaxy clusters.

\subsection{Acoustic wave dissipation and thermalization of AGN energy in cool cores}

Intermittent activity of bipolar AGN jets at the centers of cool core clusters is expected to generate sound waves in the ICM. Concentric ripples in the X-ray emissivity have been detected in the 
Perseus (Fabian et al. 2003) and Virgo clusters (Forman et al. 2005) and have been interpreted as sound waves generated by central supermassive black holes. These arcs have characteristic widths (or wavelengths) $\lambda$ of order $\sim 1$ to $10 \mathrm{kpc}$ and are seen up to several tens of kpc away from cluster centers, suggesting that the waves propagate over substantial fractions of cool core radii before completely dissipating. The recent investigation of Bambic \& Reynolds (2019) showed that up to 25-30\% of the energy injected as fast jets by the AGN can end up as sound waves, highlighting the relevance of this physics to the question of AGN feedback.

The above argument suggests that sound waves could be a promising agent responsible for converting the mechanical energy of the AGN to the thermal energy of the ICM. Appealing features of this mode of heating are that the waves provide a natural mechanism to: (i) quickly deliver AGN energy to the ICM (i.e., on sound crossing timescales that are typically shorter than radiative cooling timescales), and (ii) distribute the energy over a large fraction of the cool core volume (rather than dissipating the energy close to the AGN jet axis). These are desirable features of the model because they can help to explain why cool cores remain globally thermally stable over times comparable to the Hubble time. Furthermore, ICM heating via sound wave thermalization is a gentle process involving very subsonic velocity fluctuations. This is a particularly appealing feature of this mode of heating given recent Hitomi observations (Hitomi Collaboration et al. 2016; Fabian et al. 2017) that suggest that the ICM in the Perseus cluster is very calm.

Tapping of sound wave energy can occur via a number of mechanisms. Hydrodynamical simulations of AGN outbursts invoking Spitzer ion viscosity demonstrated that sound wave dissipation can offset radiative cooling losses and that the waves can propagate significant distances away from cluster centers (Ruszkowski et al. 2004a,b). However, the central cool core regions tend to be somewhat overheated in this model. This problem is further exacerbated by the fact that classical Spitzer conduction is $\left(m_{p} / m_{e}\right)^{1 / 2}$ more effective than Spitzer viscosity in dissipating waves. This suggests that transport processes need to be substantially suppressed in the ICM in order to eliminate tension with the observations. The necessary level of suppression was quantified using linear theory by Fabian et al. (2005b), and this work was further extended by Zweibel et al. (2018), who included 
the self-limiting nature of dissipation by electron thermal conduction, electron-ion non-equilibration effects, and provided estimates of kinetic effects by comparing to a semi-collisionless theory.

One of the main limitations of the above investigations was that the level of transport was quantified by specifying ad hoc Spitzer suppression factors. Our model allows one to relax these assumptions and it can be applied to make physically-motivated predictions for the evolution of the dissipating sound waves. In particular, our model self-consistently bridges the transition from the collisional to whistler-mediated transport regimes. Properly accounting for this transition may prove crucial for our understanding of the thermalization of the AGN-induced sound waves - while the sound wave dissipation at the very centers of cool cores may occur via collisional processes, collisionless processes are likely to dominate over a wide range of distances away from cool core centers where $L_{c} \beta>\lambda$. For example, the Coulomb mean free path is $L_{c} \sim 2 \times 10^{-3} \mathrm{kpc}$ and $\sim 4 \times 10^{-2} \mathrm{kpc}$ at the centers of the Virgo and Perseus clusters, respectively; and at the distance of $\sim 50 \mathrm{kpc}$ from the center, the corresponding values are $L_{c} \sim 0.4 \mathrm{kpc}$ and $\sim 0.2 \mathrm{kpc}$ (see, e.g., Zhuravleva et al. (2014) for density and temperature profiles in these clusters). Given typical plasma $\beta \sim 10^{2}$ in the ICM and sound wave $\lambda \sim 1$ to $10 \mathrm{kpc}$ (e.g., Fabian et al. (2006)), both the collisional and whistler regimes are expected to play a role in sound wave dissipation and propagation. Furthermore, the suppression of conduction expected in the whistler-dominated scattering regime may offer a natural explanation for the observed large propagation lengths of sound waves in the ICM. Interestingly, Kunz et al. (2020) demonstrated that suppression of collisionless Landau damping of ion acoustic waves is expected when the relative wave amplitudes exceed $2 / \beta$. Such waves could then be self-sustaining and propagate over large distances in a manner resembling sound wave propagation in a weakly collisional ICM.

An investigation of the consequences of whistler-mediated transport for the evolution of the AGNinduced waves represents an interesting future research direction, and we intend to report on the results of this investigation in future publications.

\subsection{Comparison with observations in the solar wind}


The solar wind is diffuse plasma flowing at high Mach number outward in the heliosphere from the sun. In situ satellite observations have produced enormous amounts of data on its properties and the Parker Solar Probe mission will facilitate measurements as close as $10 R_{\odot}$ with $R_{\odot}$ the solar radius. The electron temperature falls slowly with radial distance, from around $30 \mathrm{eV}$ at $35 R_{\odot}$ to $10 \mathrm{eV}$ at $120 R_{\odot}$ (Moncuquet et al. 2020). The collisionality of the solar wind depends on the plasma density and varies over a wide range, including a transition from collisional to collisionless behavior as the collisional mean free path $L_{c}$ varies from smaller than to larger than the scale length of the electron temperature gradient $L$. Measurements from the large Wind spacecraft dataset revealed that the electron heat flux rolled over to a value below the collisionless heat flux $Q_{0}$ as $L_{c} / L$ increases and the ambient plasma becomes more collisionless (Bale et al. 2013). While $\beta_{e}$ of the solar wind is nominally of order unity around $1 \mathrm{AU}$, this value is actually highly variable. The Wind dataset revealed more than $12 \mathrm{k}$ measurements of $\beta_{e}$ in the range from 5-100. An important conclusion from this dataset in light of the threshold for whistler growth in Eq. (48) is that value of $L_{c} / L$ above which the electron heat flux rolled over to a constant value decreased with higher $\beta_{e}$. An important development was the confirmation from the Wind (Tong et al. 2018) and ARTEMIS (Tong et al. 2019) datasets that the ratio of $Q / Q_{0}$ scales like $\beta_{e}^{-1}$ at high $\beta_{e}$, consistent with whistler-limited heat flux. Further, the measured amplitude of whistler waves increased both with heat flux and with $\beta_{e}$ (Tong et al. 2019), consistent with the heat flux as the whistler drive mechanism.

While the general features of the whistler wave activity and the associated heat flux measurements support the idea that heat flux driven whistlers play a role in limiting electron heat flux in the solar wind, significant uncertainties remain. The ARTEMIS observations have been interpreted as "quasi-parallel" whistler waves (Tong et al. 2019). The PIC simulations as well as analytic analysis, however, have established that parallel whistlers are not capable of significantly limiting electron heat flux since the electrons carrying the dominant heat flux do not resonate with parallel whistlers (Roberg-Clark et al. 2016; Komarov et al. 2018). On the other hand, the magnetic field measurements are limited to the spin plane of the spacecraft so no direct measurements of the direction of the wavevectors of the whistlers was possible. The amplitude of measured whistlers in the solar 
wind was small, around $2 \%$ of the ambient magnetic field, leading to concern that the whistler waves were too small in amplitude to limit electron thermal transport. On the other hand, the saturated level of fluctuations given below Eq. (14), $\varepsilon_{w} \sim 10\left(\rho_{e} / L\right) n T$, is very small for the realistic values of $\rho_{e} / L$ of the solar wind. For $T_{e} \sim 10 \mathrm{eV}$ and $B_{0} \sim 10^{-4} \mathrm{G}, \rho_{e} \sim 1 \mathrm{~km}$. Taking a temperature scale length of around $100 R_{\odot} \sim 10^{5} \mathrm{~km}$ and $\beta_{e} \sim 10$, the predicted whistler fluctuation level $\delta B / B_{0} \sim 1 \%$, which is in the range of the observations. The scaling $Q / Q_{0} \sim \beta_{e}^{-1}$ at high $\beta_{e}$ is now firmly established (Tong et al. 2018, 2019). There has been no mechanism other than heat flux limited whistlers proposed to explain this scaling.

The more recent measurements from the PSP mission have further established the role of whistlers in limiting the heat flux in the solar wind and specifically scattering the field aligned electron strahl, which has energies in the range of $100 \mathrm{eV}$ to $1 \mathrm{keV}$, into the more isotropic halo distribution (Agapitov et al. 2020; Cattell et al. 2021a,b). The presence of large amplitude whistlers was correlated with local regions of increased plasma $\beta$ (Agapitov et al. 2020; Cattell et al. 2021a). The presence of whistlers also correlated well with thresholds of fan instability, which is an oblique whistler driven by the $n=-1$ resonance (Vasko et al. 2019; Cattell et al. 2021a). Further, the pitch angle width of the measured strahl electrons as also linked to the presence or absence of large amplitude whistlers, with the pitch angle width increasing with the strength of whistler wave activity, establishing that strahl scattering is caused by resonant interactions with whistlers.

\section{ACKNOWLEDGMENTS}

The authors thank the KITP program Multiscale Phenomena in Plasma Astrophysics for an inspiring hospitality that fostered the ideas for this work. C.P. thanks Thomas Berlok for valuable comments on the manuscript and enlightening discussions. This research was supported in part by the National Science Foundation under Grant No. NSF PHY-1748958. J.F.D., M.S. and A.E. acknowledge support from NASA ATP Grant No. NNX17AG27G, NASA Grant No. 80NNSC19K0848 and NSF Grant No. PHY1805829. C.P. and T.T. acknowledge support by the European Research 
Table 1.

\begin{tabular}{|c|c|c|c|c|c|}
\hline \multirow[t]{2}{*}{ 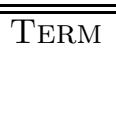 } & \multirow[t]{2}{*}{ "DEFINITION } & \multicolumn{2}{|l|}{ WHEN $\nu_{e i}^{e} \gg \nu_{w e}$} & \multicolumn{2}{|l|}{$\overline{\text { WHEN } \nu_{e i}^{e}=0}$} \\
\hline & & General & $\gamma=\frac{4}{3}$ & General & $\gamma=\frac{4}{3}$ \\
\hline (1) & $(2)$ & $(3)$ & $(4)$ & $(5)$ & $(6)$ \\
\hline$\alpha_{1}$ & $\frac{2}{3}\left\langle\frac{m v^{2}}{2 T_{0}} \frac{\nu_{e}}{\nu}\right\rangle$ & $\frac{4}{3 \sqrt{\pi}} \Gamma(4)$ & 4.51 & $\frac{4}{3 \sqrt{\pi}} \Gamma\left(\frac{5-\gamma}{2}\right)$ & 0.708 \\
\hline$\alpha_{2}$ & $\frac{2}{3}\left\langle\frac{m v^{2}}{2 T_{0}}\left(\frac{m v^{2}}{2 T_{0}}-\frac{5}{2}\right) \frac{\nu_{e}}{\nu}\right\rangle$ & $\frac{2}{\sqrt{\pi}} \Gamma(4)$ & 6.77 & $-\frac{2}{3 \sqrt{\pi}} \gamma \Gamma\left(\frac{5-\gamma}{2}\right)$ & -0.472 \\
\hline$\alpha_{3}$ & $\frac{2}{3}\left\langle\frac{m v^{2}}{2 T_{0}} \frac{\nu_{e}}{\nu} \frac{\nu_{w}}{\nu_{w e}}\right\rangle$ & $\frac{4}{3 \sqrt{\pi}} \Gamma\left(\frac{8+\gamma}{2}\right)$ & 11.1 & 1 & 1 \\
\hline$\alpha_{4}$ & $\frac{2}{3}\left\langle\left(\frac{m v^{2}}{2 T_{0}}\right)^{2} \frac{\nu_{e}}{\nu}\right\rangle$ & $\frac{4}{3 \sqrt{\pi}} \Gamma(5)$ & 18.1 & $\frac{4}{3 \sqrt{\pi}} \Gamma\left(\frac{7-\gamma}{2}\right)$ & 1.30 \\
\hline$\alpha_{5}$ & $\frac{2}{3}\left\langle\left(\frac{m v^{2}}{2 T_{0}}\right)^{2}\left(\frac{m v^{2}}{2 T_{0}}-\frac{5}{2}\right) \frac{\nu_{e}}{\nu}\right\rangle$ & $\frac{10}{3 \sqrt{\pi}} \Gamma(5)$ & 45.1 & $\frac{4}{3 \sqrt{\pi}}\left(1-\frac{\gamma}{2}\right) \Gamma\left(\frac{7-\gamma}{2}\right)$ & 0.432 \\
\hline$\alpha_{6}$ & $\frac{2}{3}\left\langle\left(\frac{m v^{2}}{2 T_{0}}\right)^{2} \frac{\nu_{e}}{\nu} \frac{\nu_{w}}{\nu_{w e}}\right\rangle$ & $\frac{4}{3 \sqrt{\pi}} \Gamma\left(\frac{10+\gamma}{2}\right)$ & 51.6 & $\frac{5}{2}$ & 2.5 \\
\hline$\alpha_{7}$ & $\frac{2}{3}\left\langle\frac{m v^{2}}{2 T_{0}} \frac{\nu_{e}}{\nu} \frac{\nu_{w}^{2}}{\nu_{w e}^{2}}\right\rangle$ & $\frac{4}{3 \sqrt{\pi}} \Gamma(4+\gamma)$ & 30.2 & $\frac{4}{3 \sqrt{\pi}} \Gamma\left(\frac{5+\gamma}{2}\right)$ & 1.76 \\
\hline$\alpha_{8}$ & $\frac{2}{3}\left\langle\frac{m v^{2}}{2 T_{0}}\left(\frac{m v^{2}}{2 T_{0}}-\frac{5}{2}\right) \frac{\nu_{e}}{\nu} \frac{\nu_{w}}{\nu_{w e}}\right\rangle$ & $\frac{2}{3 \sqrt{\pi}}(3+\gamma) \Gamma\left(\frac{8+\gamma}{2}\right)$ & 24.0 & 0 & 0 \\
\hline$\alpha_{9}$ & $\frac{2}{3}\left\langle\frac{m v^{2}}{2 T_{0}} \frac{\nu_{w}}{\nu_{w e}}\right\rangle$ & $\frac{4}{3 \sqrt{\pi}} \Gamma\left(\frac{5+\gamma}{2}\right)$ & 1.76 & $\frac{4}{3 \sqrt{\pi}} \Gamma\left(\frac{5+\gamma}{2}\right)$ & 1.76 \\
\hline$\alpha_{10}$ & $\alpha_{8}-\frac{\alpha_{3} \alpha_{2}}{\alpha_{1}}=\alpha_{6}-\frac{\alpha_{3} \alpha_{4}}{\alpha_{1}}$ & $\frac{2}{3 \sqrt{\pi}} \gamma \Gamma\left(\frac{8+\gamma}{2}\right)$ & 7.38 & $\frac{\gamma}{2}$ & 0.667 \\
\hline$\alpha_{11}$ & $\alpha_{9}+\frac{\nu_{w e}}{\nu_{e}}\left(\frac{\alpha_{3}^{2}}{\alpha_{1}}-\alpha_{7}\right)$ & $\frac{4}{3 \sqrt{\pi}} \Gamma\left(\frac{5+\gamma}{2}\right)$ & 1.76 & $\Gamma\left(\frac{5}{2}\right) / \Gamma\left(\frac{5-\gamma}{2}\right)$ & 1.41 \\
\hline$\alpha_{12}$ & $\alpha_{5}-\frac{\alpha_{2}}{\alpha_{1}}$ & $\frac{10}{3 \sqrt{\pi}} \Gamma(5)-\frac{3}{2}$ & 43.6 & $\left(1-\frac{\gamma}{2}\right) \Gamma\left(\frac{7-\gamma}{2}\right) / \Gamma\left(\frac{5}{2}\right)+\frac{\gamma}{2}$ & 1.10 \\
\hline$\alpha_{13}$ & $\alpha_{7}-\frac{\alpha_{3}^{2}}{\alpha_{1}}$ & $\frac{4}{3 \sqrt{\pi}}\left(\Gamma(4+\gamma)-\Gamma^{2}\left(\frac{8+\gamma}{2}\right) / 6\right)$ & 3.05 & $\frac{4}{3 \sqrt{\pi}} \Gamma\left(\frac{5+\gamma}{2}\right)-\frac{3 \sqrt{\pi}}{4} \Gamma^{-1}\left(\frac{5-\gamma}{2}\right)$ & 0.351 \\
\hline
\end{tabular}

Note-A table of the dimensionless parameters defining the electron transport equations. The brackets denote an average over a 3D Maxwellian distribution with temperature $T_{0}$. The parameters are evaluated in the limits when classical scattering dominates whistler scattering $\left(\nu_{e i}^{e} \gg \nu_{w e}\right)$ where $\nu \propto v^{-3}$ and when whistler scattering dominates classical scattering $\left(\nu_{w e} \gg \nu_{e i}^{e}\right)$ where $\nu \propto v^{\gamma}$. Numerical values are given for the limits when $\gamma=4 / 3$.

Council (ERC) under ERC-CoG grant CRAGSMAN-646955. C.S.R. acknowledges support by the ERC under ERC-Adv grant DISKtoHALO-834203. M.R. acknowledges support from NSF grants AST 1715140 and AST 2009227.

\section{APPENDIX}

\section{A. GLOBAL COUPLED MHD AND ELECTRON TRANSPORT EQUATIONS}

The transport equations presented in Sec. 2 provide a suitable framework when combined with an MHD description to describe the large-scale dynamics of the ICM system. However, for simplicity 
the equations were discussed in the context of a unidirectional temperature gradient with whistler waves propagating in a single direction down the gradient. In a real system the temperature will develop complex structure that will produce whistlers propagating in both directions with respect to the magnetic field. Thus, a set of equations that can be used to describe the full dynamics of a system with an arbitrary temperature structure must include bi-directional whistler waves and their interaction with the ambient temperature gradient. We therefore generalize the equations presented in Sec. 2 to describe whistlers propagating in both directions with respect to the local magnetic field in three-dimensional space. The generalization is straightforward except for the addition of a cross term arising from $C_{1}\left(f_{1}\right)$ in Eq. (31). The cross term describes electron heating associated with the interaction of counter-streaming whistler waves. This has been interpreted as second order Fermi acceleration in the case of cosmic ray transport (Thomas \& Pfrommer 2019). The generalized equations when combined with MHD are suitable for describing the dynamics of a system with arbitrary gradients and arbitrary classical collision rates. We use the Lorentz-Heaviside system of units throughout this Appendix and denote the dyadic product of any two vectors $\mathbf{P}$ and $\mathbf{Q}$ by $\mathbf{P Q}$.

\section{A.1. Derivation of the wave energy equation}

For the sake of transparency of this derivation, we suppress the source terms in the whistler wave equation, namely the whistler wave growth and loss terms (due to drag and second-order Fermi acceleration). Moreover, in this derivation we only consider whistler waves that move in one direction and generalize the result at the end of this section to also account for backwards moving whistlers.

The energy equation for electromagnetic fields is:

$$
\frac{\partial\left(\mathbf{E}^{2} / 2+\mathbf{B}^{2} / 2\right)}{\partial t}+c \nabla \cdot(\mathbf{E} \times \mathbf{B})+\mathbf{J} \cdot \mathbf{E}=0,
$$

where the electric field in Hall-MHD (which is the appropriate limit) is given by:

$$
\mathbf{E}+\frac{[\mathbf{U}-\mathbf{J} /(n e)] \times \mathbf{B}}{c}=\mathbf{0} .
$$


The Hall term, which is proportional to $\mathbf{J} /(n e)$, is assumed to be zero in standard MHD. This term does not do any work on the electro-magnetic field as can be shown:

$$
\begin{aligned}
\mathbf{J} \cdot \mathbf{E} & =-\mathbf{J} \cdot \frac{[\mathbf{U}-\mathbf{J} /(n e)] \times \mathbf{B}}{c}=-\mathbf{J} \cdot \frac{\mathbf{U} \times \mathbf{B}}{c}=\mathbf{U} \cdot \frac{\mathbf{J} \times \mathbf{B}}{c} \\
& =\mathbf{U} \cdot[(\nabla \times \mathbf{B}) \times \mathbf{B}]=-\mathbf{U} \cdot\left[\nabla \cdot\left(\frac{\mathbf{B}^{2}}{2} \mathbf{1}-\mathbf{B B}\right)\right]
\end{aligned}
$$

where we use vector identities in the last step and assumed a divergence-free magnetic field.

We are interested in the energy transport of small-amplitude whistler waves. To this end, we perturb the energy equation in Eq. (A1) assuming small deviations of the electric and magnetic fields from its mean values. Carrying out a perturbation analysis and neglecting the energy density of the electric field in the energy equation yields:

$$
\frac{\partial \delta \mathbf{B}^{2} / 2}{\partial t}+c \nabla \cdot(\delta \mathbf{E} \times \delta \mathbf{B})+\delta \mathbf{J} \cdot \delta \mathbf{E}=0,
$$

and the perturbed electric field for whistler waves is given by:

$$
\delta \mathbf{E}+\frac{\left(\mathbf{U}+\mathbf{V}_{w}\right) \times \delta \mathbf{B}}{c}=\mathbf{0},
$$

where $\mathbf{V}_{w}$ is the whistler wave velocity. Inserting the perturbed electric field into the divergence terms and expanding the double-cross product yields:

$$
\frac{\partial \delta \mathbf{B}^{2} / 2}{\partial t}-\nabla \cdot\left[\left(\mathbf{U}+\mathbf{V}_{w}\right) \cdot \delta \mathbf{B} \delta \mathbf{B}-\delta \mathbf{B}^{2}\left(\mathbf{U}+\mathbf{V}_{w}\right)\right]+\delta \mathbf{J} \cdot \delta \mathbf{E}=0
$$

The magnetic field tensor is given by:

$$
\delta \mathbf{B} \delta \mathbf{B}=\left[\begin{array}{ccc}
\delta B_{x} \delta B_{x} & \delta B_{y} \delta B_{x} & 0 \\
\delta B_{x} \delta B_{y} & \delta B_{y} \delta B_{y} & 0 \\
0 & 0 & 0
\end{array}\right]
$$

where we assumed that the whistler waves are propagating quasi-parallel to the mean magnetic field and evaluated the tensor in a coordinate system where this mean magnetic field is locally aligned with the $z$ axis. In general the whistler wave has some phase. As we have no a priori information 
about this phase, we take an average of the magnetic field tensor over all possible phases and assume an equal probability for all phases. This results in

$$
\langle\delta \mathbf{B} \delta \mathbf{B}\rangle=\left[\begin{array}{ccr}
\delta \mathbf{B}^{2} / 2 & 0 & 0 \\
0 & \delta \mathbf{B}^{2} / 2 & 0 \\
0 & 0 & 0
\end{array}\right]=\frac{\delta \mathbf{B}^{2}}{2}(\mathbf{1}-\mathbf{b b}),
$$

where \langle\rangle denotes the ensemble average, $\delta B_{x}=|\delta \mathbf{B}| \cos (\phi), \delta B_{y}= \pm|\delta \mathbf{B}| \sin (\phi), \mathbf{b}=\mathbf{B} /|\mathbf{B}|$ is the unit vector along $\mathbf{B}$, and we applied the ergodic theorem so that we effectively replace ergodic averaging by phase averaging. ${ }^{2}$ Taking the ensemble average of Eq. (A7) and inserting the result of Eq. (A9) yields:

$$
\frac{\partial \delta \mathbf{B}^{2} / 2}{\partial t}-\nabla \cdot\left[(\mathbf{1}-\mathbf{b b}) \cdot\left(\mathbf{U}+\mathbf{V}_{w}\right) \frac{\delta \mathbf{B}^{2}}{2}-\delta \mathbf{B}^{2}\left(\mathbf{U}+\mathbf{V}_{w}\right)\right]+\langle\delta \mathbf{J} \cdot \delta \mathbf{E}\rangle=0
$$

After noting that $(\mathbf{1}-\mathbf{b b}) \cdot \mathbf{V}_{w}=0$ we get:

$$
\frac{\partial \delta \mathbf{B}^{2} / 2}{\partial t}+\nabla \cdot\left[\delta \mathbf{B}^{2}\left(\mathbf{U}+\mathbf{V}_{w}\right)-(\mathbf{1}-\mathbf{b b}) \cdot \mathbf{U} \frac{\delta \mathbf{B}^{2}}{2}\right]+\langle\delta \mathbf{J} \cdot \delta \mathbf{E}\rangle=0 .
$$

The work done on the perturbed electro-magetic field is:

$$
\delta \mathbf{J} \cdot \delta \mathbf{E}=-\mathbf{U} \cdot\left[\nabla \cdot\left(\frac{\delta \mathbf{B}^{2}}{2} \mathbf{1}-\delta \mathbf{B} \delta \mathbf{B}\right)\right],
$$

or, after taking the ensemble average (using Eq. A9),

$$
\langle\delta \mathbf{J} \cdot \delta \mathbf{E}\rangle=-\mathbf{U} \cdot\left[\nabla \cdot\left(\frac{\delta \mathbf{B}^{2}}{2} \mathbf{b b}\right)\right] .
$$

Inserting this expression into Eq. (A11) yields:

$$
\frac{\partial \delta \mathbf{B}^{2} / 2}{\partial t}+\nabla \cdot\left[\frac{\delta \mathbf{B}^{2}}{2} \mathbf{U}+\frac{\delta \mathbf{B}^{2}}{2} \mathbf{b b} \cdot \mathbf{U}+\delta \mathbf{B}^{2} \mathbf{V}_{w}\right]=\mathbf{U} \cdot\left[\nabla \cdot\left(\frac{1}{2} \delta \mathbf{B}^{2} \mathbf{b b}\right)\right] .
$$

Defining the energy density and pressure tensor of whistler waves, respectively,

$$
\begin{aligned}
\varepsilon_{w} & =\frac{\delta \mathbf{B}^{2}}{2}, \text { and } \\
\mathbf{P}_{w} & =\frac{\delta \mathbf{B}^{2}}{2} \mathbf{b b}
\end{aligned}
$$

${ }^{2}$ Note that $1 /(2 \pi) \int_{-\pi}^{\pi} d \phi \sin ^{2}(\phi)=1 /(2 \pi) \int_{-\pi}^{\pi} d \phi \cos ^{2}(\phi)=1 / 2$. 
simplifies the notation of Eq. (A14):

$$
\frac{\partial \varepsilon_{w}}{\partial t}+\nabla \cdot\left[\left(\varepsilon_{w} \mathbf{1}+\mathbf{P}_{w}\right) \cdot \mathbf{U}+2 \varepsilon_{w} \mathbf{V}_{w}\right]=\mathbf{U} \cdot\left[\nabla \cdot \mathbf{P}_{w}\right]
$$

In order to generalize this result to forward and backward propagating whistlers, we define the corresponding energy densities, $\varepsilon_{w}^{ \pm}$, and wave pressure tensor, $\mathbf{P}_{w}^{ \pm}$, add the whistler source terms to the right-hand side, and obtain ${ }^{3}$ :

$$
\frac{\partial \varepsilon_{w}^{ \pm}}{\partial t}+\nabla \cdot\left[\left(\varepsilon_{w}^{ \pm} \mathbf{1}+\mathbf{P}_{w}^{ \pm}\right) \cdot \mathbf{U} \pm 2 \varepsilon_{w}^{ \pm} \mathbf{V}_{w}\right]=\mathbf{U} \cdot\left[\nabla \cdot \mathbf{P}_{w}^{ \pm}\right]+G_{w}^{ \pm}-H_{w}^{ \pm} .
$$

The superscript \pm denotes waves propagating down and up the temperature gradient, respectively. The whistler energy density and pressure tensor are given by

$$
\begin{aligned}
\varepsilon_{w}^{ \pm} & =\frac{\delta \mathbf{B}_{ \pm}^{2}}{2} \text {, and } \\
\mathbf{P}_{w}^{ \pm} & =\frac{\delta \mathbf{B}_{ \pm}^{2}}{2} \mathbf{b b} .
\end{aligned}
$$

The whistler growth term, $G_{w}^{ \pm}$, and the whistler loss term, $H_{w}^{ \pm}$, are given by

$$
\begin{aligned}
& G_{w}^{ \pm}= \pm V_{\mathrm{st}}^{ \pm} n k_{\mathrm{B}} \mathbf{b} \cdot \nabla T \\
& H_{w}^{ \pm}=m_{e} n V_{w}^{2}\left(\alpha_{11} \nu_{w e}^{ \pm}+\alpha_{13} \frac{\nu_{w e}^{+} \nu_{w e}^{-}}{\nu_{e}}\right),
\end{aligned}
$$

where $k_{\mathrm{B}}$ is Boltzmann's constant. The first term in the whistler loss term $H_{w}^{ \pm}$is associated with the wave drag on the electrons that causes them to be heated and includes contributions from whistlers propagating in both directions. The term proportional to the product of the scattering rates of bi-direction whistlers corresponds to second order Fermi acceleration. The generalized electron streaming velocities $V_{\mathrm{st}}^{ \pm}$with the forward and backward propagating whistler waves are defined as

$$
V_{\mathrm{st}}^{ \pm}=V_{w} \alpha_{10} \frac{\nu_{w e}^{ \pm}}{\nu_{e}}
$$

where $V_{w}=\mathbf{V}_{w} \cdot \mathbf{b}=v_{t e} / \beta_{e}$ is the non-directional whistler phase speed.

$$
\begin{gathered}
{ }^{3} \text { Using the identity } \nabla \cdot\left[\mathbf{P}_{w}^{ \pm} \cdot \mathbf{U}\right]-\mathbf{U} \cdot\left[\nabla \cdot \mathbf{P}_{w}^{ \pm}\right]=\mathbf{P}_{w}^{ \pm}: \nabla \mathbf{U} \text {, this equation can also be written } \\
\frac{\partial \varepsilon_{w}^{ \pm}}{\partial t}+\nabla \cdot\left[\varepsilon_{w}^{ \pm} \mathbf{U} \pm 2 \varepsilon_{w}^{ \pm} \mathbf{V}_{w}\right]=-\mathbf{P}_{w}^{ \pm}: \nabla \mathbf{U}+G_{w}^{ \pm}-H_{w}^{ \pm}
\end{gathered}
$$




\section{A.2. Momentum conservation and kinetic energy equation}

Analogously, it is possible to perturb the momentum equation to account for the forces exerted by the whistler waves. We start with the momentum equation for the composite ion-electron fluid, which is given by

$$
\rho \frac{d \mathbf{U}}{d t}+\nabla P_{\text {th }}=\frac{\mathbf{J} \times \mathbf{B}}{c}=(\nabla \times \mathbf{B}) \times \mathbf{B}=-\nabla \cdot\left(\frac{\mathbf{B}^{2}}{2} \mathbf{1}-\mathbf{B B}\right),
$$

where where $d / d t=\partial / \partial t+\mathbf{U} \cdot \nabla$ is the Lagrangian derivative, $P_{\mathrm{th}}=P_{e}+P_{i}$ is the total (ion plus electron) pressure, and we used $\mathbf{J}=c \nabla \times \mathbf{B}$ (neglecting the displacement current in the HallMHD approximation). Introducing the perturbations in the magnetic field while neglecting linear contributions due to ensemble averaging yields:

$$
\rho \frac{d \mathbf{U}}{d t}+\nabla \cdot\left(P_{\mathrm{th}} \mathbf{1}+\frac{\mathbf{B}^{2}}{2} \mathbf{1}-\mathbf{B B}+\frac{\delta \mathbf{B}^{2}}{2} \mathbf{1}-\langle\delta \mathbf{B} \delta \mathbf{B}\rangle\right)=\mathbf{0},
$$

which can be simplified using Eq. (A9) to yield:

$$
\rho \frac{d \mathbf{U}}{d t}+\nabla \cdot\left(P_{\mathrm{th}} \mathbf{1}+\frac{\mathbf{B}^{2}}{2} \mathbf{1}-\mathbf{B B}+\frac{\delta \mathbf{B}^{2}}{2} \mathbf{b b}\right)=\mathbf{0} .
$$

Using the definitions for the wave energy and wave pressure while accounting for the two wave types and inserting the continuity equation yields the final conservative form of the momentum equation in the presence of whistler waves:

$$
\frac{\partial \rho \mathbf{U}}{\partial t}+\nabla \cdot\left(\rho \mathbf{U U}+P_{\mathrm{th}} \mathbf{1}+\frac{\mathbf{B}^{2}}{2} \mathbf{1}-\mathbf{B B}+\mathbf{P}_{w}^{+}+\mathbf{P}_{w}^{-}\right)=\mathbf{0} .
$$

Multiplying this equation by $\mathbf{U}$ and using the continuity equation yields the kinetic energy equation:

$$
\frac{\partial \varepsilon_{\text {kin }}}{\partial t}+\nabla \cdot\left(\varepsilon_{\text {kin }} \mathbf{U}\right)=-\mathbf{U} \cdot\left\{\nabla \cdot\left[\left(P_{\mathrm{th}}+\frac{\mathbf{B}^{2}}{2}\right) \mathbf{1}-\mathbf{B B}+\mathbf{P}_{w}^{+}+\mathbf{P}_{w}^{-}\right]\right\} .
$$

where $\varepsilon_{\text {kin }}=\rho \mathbf{U}^{2} / 2$.

\section{A.3. Magnetic energy equation}

The magnetic induction law is given by,

$$
\frac{\partial \mathbf{B}}{\partial t}+\nabla \cdot(\mathbf{B U}-\mathbf{U B})=\mathbf{0}
$$


Multiplying this equation by $\mathbf{B}$ yields the equation for the magnetic energy:

$$
\frac{\partial \varepsilon_{B}}{\partial t}+\nabla \cdot\left[\mathbf{U} \cdot\left(\varepsilon_{B}+\frac{\mathbf{B}^{2}}{2}\right)-(\mathbf{U} \cdot \mathbf{B}) \mathbf{B}\right]=\mathbf{U} \cdot\left\{\nabla \cdot\left[\frac{\mathbf{B}^{2}}{2} \mathbf{1}-\mathbf{B B}\right]\right\},
$$

where $\varepsilon_{B}=\mathbf{B}^{2} / 2$.

\section{A.4. Ion and electron energy equations}

The ion and electron energy equations are

$$
\begin{gathered}
\frac{\partial \varepsilon_{i}}{\partial t}+\nabla \cdot\left[\mathbf{U}\left(\varepsilon_{i}+P_{i}\right)\right]=\mathbf{U} \cdot \nabla P_{i}-\frac{\varepsilon_{i}-\varepsilon_{e}}{\tau_{\mathrm{eq}}} \\
\frac{\partial \varepsilon_{e}}{\partial t}+\nabla \cdot\left[\mathbf{U}\left(\varepsilon_{e}+P_{e}\right)+\mathbf{Q}_{e, \mathrm{st}}+\mathbf{Q}_{e, \mathrm{dif}}\right]=\mathbf{U} \cdot \nabla P_{e}-\frac{\varepsilon_{e}-\varepsilon_{i}}{\tau_{\mathrm{eq}}}+\sum_{ \pm}\left(H_{w}^{ \pm}-G_{w}^{ \pm}\right) .
\end{gathered}
$$

where $\varepsilon_{i}=3 / 2 n k_{\mathrm{B}} T_{i}$ and $\varepsilon_{e}=3 / 2 n k_{\mathrm{B}} T_{e}$ are the energy densities of ions and electrons, respectively, and the electron streaming and diffusion fluxes are given by

$$
\begin{aligned}
\mathbf{Q}_{e, \mathrm{st}} & =\left(V_{\mathrm{st}}^{+}-V_{\mathrm{st}}^{-}\right) P_{e} \mathbf{b}, \\
\mathbf{Q}_{e, \mathrm{dif}} & =-\kappa_{e} \mathbf{b b} \cdot \nabla T_{e} .
\end{aligned}
$$

While the ion energy equation is standard, the equation for the electron energy density is the same as given in Eq. (4) but generalized for the general fluid velocity $\mathbf{U}$ and the whistler streaming velocities $V_{\mathrm{st}}^{ \pm}$. The energy equilibration time between electrons and ions is denoted by $\tau_{\mathrm{eq}}$, which is typically longer by $\sqrt{m_{i} / m_{e}}$ than the classical electron-ion scattering time. The whistler waves can also transfer energy between electrons and ions but the detailed scaling behavior for this transfer has not been established (Roberg-Clark et al. 2018b). Viscous terms could also be included in the momentum and ion pressure equations. The parallel conductivity $\kappa_{e}$ is

$$
\kappa_{e}=\alpha_{12} \frac{n k_{\mathrm{B}} T_{e}}{m_{e} \nu_{e}} .
$$

The total scattering rate $\nu_{e}=\nu_{e i}^{e}+\nu_{w e}^{+}+\nu_{w e}^{-}$is the sum of $\nu_{e i}^{e}$, the classical electron-ion scattering

rate, and the scattering rates $\nu_{w e}^{ \pm}$from the forward and backward propagating whistlers, all evaluated at the electron thermal velocity $v_{t e}$,

$$
\nu_{e i}^{e}=\frac{4 \pi e^{4} n \Lambda}{m_{e}^{2} v_{t e}^{3}}, \quad \nu_{w e}^{ \pm}=0.1 \Omega_{e} \frac{\varepsilon_{w}^{ \pm}}{\varepsilon_{B}},
$$


where $\varepsilon_{w}^{ \pm}$and $\varepsilon_{B}=B^{2} / 8 \pi$ are the whistler and magnetic energy densities and $\Lambda$ is the Coulomb logarithm.

The total electron heat flux $\mathbf{Q}_{e}=\mathbf{U}\left(\varepsilon_{e}+P_{e}\right)+\mathbf{Q}_{e, \text { st }}+\mathbf{Q}_{e, \text { dif }}$ is composed of the flux due to advection of electron enthalpy, the effective streaming flux due to electron-whistler wave scattering, as well as the diffusive flux. In Eq. (A33), the forward and backward propagating whistlers try to carry the electron energy in opposite directions along $\mathbf{B}$. The wave with the dominant scattering rate wins out and as a result the direction of advection can change sign with the ambient parallel temperature gradient.

\section{A.5. Full set of equations of whistler $M H D$}

For completeness, here we summarize the complete set of equations for whistler MHD that constitute a complete description of plasma dynamics in the high $\beta$ ICM (to which a description of anisotropic viscosity can be added):

$$
\begin{aligned}
\frac{\partial \rho}{\partial t}+\nabla \cdot(\rho \mathbf{U}) & =0 \\
\frac{\partial \rho \mathbf{U}}{\partial t}+\nabla \cdot\left[\rho \mathbf{U} \mathbf{U}+\left(P_{\mathrm{th}}+\frac{\mathbf{B}^{2}}{2}\right) \mathbf{1}-\mathbf{B B}+\mathbf{P}_{w}^{+}+\mathbf{P}_{w}^{-}\right] & =\mathbf{0} \\
\frac{\partial \mathbf{B}}{\partial t}+\nabla \cdot(\mathbf{B} \mathbf{U}-\mathbf{U B}) & =\mathbf{0} \\
\frac{\partial \varepsilon_{i}}{\partial t}+\nabla \cdot\left[\mathbf{U}\left(\varepsilon_{i}+P_{i}\right)\right] & =\mathbf{U} \cdot \nabla P_{i}-\frac{\varepsilon_{i}-\varepsilon_{e}}{\tau_{\mathrm{eq}}} \\
\frac{\partial \varepsilon_{e}}{\partial t}+\nabla \cdot\left[\mathbf{U}\left(\varepsilon_{e}+P_{e}\right)+\mathbf{Q}_{e, \mathrm{st}}+\mathbf{Q}_{e, \mathrm{dif}}\right] & =\mathbf{U} \cdot \nabla P_{e}-\frac{\varepsilon_{e}-\varepsilon_{i}}{\tau_{\mathrm{eq}}}+\sum_{ \pm}\left(H_{w}^{ \pm}-G_{w}^{ \pm}\right) \\
\frac{\partial \varepsilon_{w}^{ \pm}}{\partial t}+\nabla \cdot\left[\left(\varepsilon_{w}^{ \pm} \mathbf{1}+\mathbf{P}_{w}^{ \pm}\right) \cdot \mathbf{U} \pm 2 \varepsilon_{w}^{ \pm} \mathbf{V}_{w}\right] & =\mathbf{U} \cdot\left[\nabla \cdot \mathbf{P}_{w}^{ \pm}\right]+G_{w}^{ \pm}-H_{w}^{ \pm}
\end{aligned}
$$

where $P_{\mathrm{th}}=P_{i}+P_{e}$ and the equations of state are given by

$$
\begin{aligned}
& P_{i}=n k_{\mathrm{B}} T_{i}=(\Gamma-1) \varepsilon_{i}, \\
& P_{e}=n k_{\mathrm{B}} T_{e}=(\Gamma-1) \varepsilon_{e},
\end{aligned}
$$


where $\Gamma=5 / 3$. The whistler growth term, $G_{w}^{ \pm}$, and the whistler loss terms, $H_{w}^{ \pm}$, (due to drag and second-order Fermi acceleration) are given by

$$
\begin{aligned}
& G_{w}^{ \pm}= \pm V_{\mathrm{st}}^{ \pm} n k_{\mathrm{B}} \mathbf{b} \cdot \nabla T_{e} \\
& H_{w}^{ \pm}=m_{e} n V_{w}^{2}\left(\alpha_{11} \nu_{w e}^{ \pm}+\alpha_{13} \frac{\nu_{w e}^{+} \nu_{w e}^{-}}{\nu_{e}}\right) .
\end{aligned}
$$

The whistler energy density and pressure tensor are given by

$$
\begin{aligned}
\varepsilon_{w}^{ \pm} & =\frac{\delta \mathbf{B}_{ \pm}^{2}}{2} \\
\mathbf{P}_{w}^{ \pm} & =\frac{\delta \mathbf{B}_{ \pm}^{2}}{2} \mathbf{b b} .
\end{aligned}
$$

The parameters $\alpha_{i}$ with various subscripts in Eqs. (A42)-(A47) are given in Table 1. As shown in the Table these parameters have simple analytic forms in the limit of large or small classical collisions but no simple analytic form for arbitrary $\nu_{w e} / \nu_{e i}^{e}$. The parameter $\gamma$ in the Table controls the dependence of $\nu_{w}$ on velocity, $\nu_{w}=\nu_{w e}\left(v / v_{t e}\right)^{\gamma}$, where, as discussed in Sec. 3, our best estimate is that $\gamma=4 / 3$. The values of the parameters $\alpha_{i}$ in the two collisionality limits have been explicitly evaluated for $\gamma=4 / 3$. A connection formula for the two collisionality limits could be constructed in a numerical implementation of the transport equations. Further, as mentioned previously the thermal conduction in Eq. (A36) does not reduce to the Spitzer value because we have discarded electron-electron collisions. The correct Spitzer value is obtained by setting $\alpha_{12}$ to 4.25 rather than the value given in Table 1.

Finally, we note that in a large-scale system in which the transport time scale $L / V_{w}$ is long compared the electron-ion energy exchange time $\tau_{\text {eq }}$, Eqs. (A41) and (A41) can be combined into a single energy equation for electrons and ions.

\section{A.6. Energy conservation}

Adding up Eqs. (A19), (A29), (A31), (A32), and (A33), we obtain total energy conservation,

$$
\frac{\partial \varepsilon_{\mathrm{tot}}}{\partial t}+\nabla \cdot\left[\mathbf{U} \cdot\left(\varepsilon_{\mathrm{tot}} \mathbf{1}+\mathbf{P}_{\mathrm{tot}}\right)-(\mathbf{U} \cdot \mathbf{B}) \mathbf{B}+2 \mathbf{V}_{w}\left(\varepsilon_{w}^{+}-\varepsilon_{w}^{-}\right)+\mathbf{Q}_{e, \mathrm{st}}+\mathbf{Q}_{e, \mathrm{dif}}\right]=0
$$


where

$$
\begin{aligned}
& \varepsilon_{\mathrm{tot}}=\varepsilon_{i}+\varepsilon_{e}+\varepsilon_{\mathrm{kin}}+\varepsilon_{B}+\varepsilon_{w}^{+}+\varepsilon_{w}^{-}, \text {and } \\
& \mathbf{P}_{\mathrm{tot}}=\left(P_{i}+P_{e}+\frac{\mathbf{B}^{2}}{2}\right) \mathbf{1}+\mathbf{P}_{w}^{+}+\mathbf{P}_{w}^{-}
\end{aligned}
$$

are the total energy density and the pressure tensor, respectively. 


\section{REFERENCES}

Agapitov, O. V., Wit, T. D. d., Mozer, F. S., et al. 2020, ApJ Lett., 891, L20, doi: $10.3847 / 2041-8213 / a b 799$ c

Arnold, H., Drake, J., Swisdak, M., \& Dahlin, J. 2019, arXiv e-prints, arXiv:1907.07554. https://arxiv.org/abs/1907.07554

Balbus, S. A. 2000, ApJ, 534, 420, doi: $10.1086 / 308732$

Balbus, S. A., \& Reynolds, C. S. 2010, ApJL, 720, L97, doi: 10.1088/2041-8205/720/1/L97

Bale, S. D., Pulupa, M., Salem, C., Chen, C. H. K., \& Quataert, E. 2013, ApJL, 769, L22, doi: 10.1088/2041-8205/769/2/L22

Bambic, C. J., \& Reynolds, C. S. 2019, ApJ, 886, 78, doi: 10.3847/1538-4357/ab4daf

Berlok, T., Quataert, E., Pessah, M. E., \&

Pfrommer, C. 2021, MNRAS, 504, 3435, doi: 10.1093/mnras/stab832

Binney, J., \& Cowie, L. L. 1981, APJ, 247, 464, doi: $10.1086 / 159055$

Biskamp, D., Schwarz, E., Zeiler, A., Celani, A., \& Drake, J. F. 1999, Physics of Plasmas, 6, 751, doi: $10.1063 / 1.873312$

Braginskii, S. I. 1965, Rev. Plasma Phys., 1, 205

Bregman, J. N., \& David, L. P. 1988, ApJ, 326, 639, doi: 10.1086/166122

Broderick, A. E., Chang, P., \& Pfrommer, C. 2012, ApJ, 752, 22, doi: 10.1088/0004-637X/752/1/22 Cattell, C., Short, B., Breneman, A., et al. 2021a, A\& A, 650, A8, doi: 10.1051/0004-6361/202039550
Cattell, C., Breneman, A., Dombeck, J., et al. 2021b, ApJL, 911, L29, doi: 10.3847/2041-8213/abefdd

Cavagnolo, K. W., Donahue, M., Voit, G. M., \& Sun, M. 2008, ApJL, 683, L107, doi: $10.1086 / 591665$

Churazov, E., Forman, W., Jones, C., \& Böhringer, H. 2000, A\& A, 356, 788. https://arxiv.org/abs/astro-ph/0002375

Drake, J. F., Arnold, H., Swisdak, M., \& Dahlin, J. T. 2019, Phys. Plasmas, 26, 012901

Fabian, A. C. 1994, ARA\&A, 32, 277, doi: 10.1146/annurev.aa.32.090194.001425

Fabian, A. C., Reynolds, C. S., Taylor, G. B., \& Dunn, R. J. H. 2005a, MNRAS, 363, 891, doi: 10.1111/j.1365-2966.2005.09484.x

—. 2005b, MNRAS, 363, 891, doi: 10.1111/j.1365-2966.2005.09484.x

Fabian, A. C., Sanders, J. S., Allen, S. W., et al. 2003, MNRAS, 344, L43, doi: 10.1046/j.1365-8711.2003.06902.x

Fabian, A. C., Sanders, J. S., Taylor, G. B., et al. 2006, MNRAS, 366, 417, doi: 10.1111/j.1365-2966.2005.09896.x

Fabian, A. C., Walker, S. A., Russell, H. R., et al. 2017, MNRAS, 464, L1, doi: $10.1093 / \mathrm{mnrasl} / \mathrm{slw} 170$

Field, G. B. 1965, ApJ, 142, 531, doi: $10.1086 / 148317$

Forman, W., Nulsen, P., Heinz, S., et al. 2005, ApJ, 635, 894, doi: 10.1086/429746 
Gary, S. P., \& Li, H. 2000, APJ, 529, 1131, doi: $10.1086 / 308294$

Hassam, A. B. 1980, Phys. Fluids, 23, 38, doi: $10.1063 / 1.862860$

Hitomi Collaboration, Aharonian, F., Akamatsu, H., et al. 2016, Nature, 535, 117, doi: $10.1038 /$ nature18627

Kannan, R., Vogelsberger, M., Pfrommer, C., et al. 2017, ApJL, 837, L18, doi: 10.3847/2041-8213/aa624b

Karimabadi, H., Akimoto, K., Omidi, N., \& Menyuk, C. R. 1990, Physics of Fluids B, 2, 606, doi: 10.1063/1.859296

Komarov, S., Schekochihin, A. A., Churazov, E., \& Spitkovsky, A. 2018, J. Plasma Phys., 84, 905840305, doi: 10.1017/S0022377818000399

Komarov, S. V., Churazov, E. M., Kunz, M. W., \& Schekochihin, A. A. 2016, MNRAS, 460, 467, doi: 10.1093/mnras/stw963

Kulsrud, R., \& Pearce, W. P. 1969, ApJ, 156, 445, doi: $10.1086 / 149981$

Kunz, M. W. 2011, MNRAS, 417, 602, doi: 10.1111/j.1365-2966.2011.19303.x

Kunz, M. W., Bogdanović, T., Reynolds, C. S., \& Stone, J. M. 2012, ApJ, 754, 122, doi: 10.1088/0004-637X/754/2/122

Kunz, M. W., Schekochihin, A. A., \& Stone, J. M. 2014, Phys. Rev. Lett., 112, 205003, doi: 10.1103/PhysRevLett.112.205003
Kunz, M. W., Squire, J., Schekochihin, A. A., \& Quataert, E. 2020, arXiv e-prints, arXiv:2006.08940. https://arxiv.org/abs/2006.08940

Lamberts, A., Chang, P., Pfrommer, C., et al. 2015, ApJ, 811, 19, doi: 10.1088/0004-637X/811/1/19

Lee, M. A. 1982, J. Geophys. Res., 87, 5063, doi: 10.1029/JA087iA07p05063

Levinson, A., \& Eichler, D. 1992, ApJ, 387, 212, doi: 10.1086/171072

Macquart, J. P., Prochaska, J. X., McQuinn, M., et al. 2020, Nature, 581, 391, doi: 10.1038/s41586-020-2300-2

McQuinn, M. 2016, ARA\&A, 54, 313, doi: 10.1146/annurev-astro-082214-122355

Moncuquet, M., Meyer-Vernet, N., Issautier, K., et al. 2020, ApJS, 246, 44, doi: 10.3847/1538-4365/ab5a84

Navarro, J. F., Frenk, C. S., \& White, S. D. M. 1997, ApJ, 490, 493, doi: 10.1086/304888

Peterson, J. R., \& Fabian, A. C. 2006, PhR, 427, 1, doi: 10.1016/j.physrep.2005.12.007

Pfrommer, C., Springel, V., Enßlin, T. A., \& Jubelgas, M. 2006, MNRAS, 367, 113, doi: 10.1111/j.1365-2966.2005.09953.x

Pistinner, S. L., \& Eichler, D. 1998, MNRAS, 301, 49, doi: 10.1046/j.1365-8711.1998.01770.x

Quataert, E. 2008, ApJ, 673, 758, doi: $10.1086 / 525248$ 
Reynolds, C. S., Heinz, S., \& Begelman, M. C. 2002, MNRAS, 332, 271, doi: 10.1046/j.1365-8711.2002.04724.x

Rincon, F., Schekochihin, A. A., \& Cowley, S. C. 2015, MNRAS, 447, L45, doi: 10.1093/mnrasl/slu179

Riquelme, M. A., Quataert, E., \& Verscharen, D. 2016, ApJ, 824, 123, doi: 10.3847/0004-637X/824/2/123

Roberg-Clark, G. T., Drake, J. F., Reynolds, C. S., \& Swisdak, M. 2016, ApJ Lett., 830, L9, doi: 10.3847/2041-8205/830/1/L9

—. 2018a, Physical Review Letters, 120, 035101, doi: 10.1103/PhysRevLett.120.035101

Roberg-Clark, G. T., Drake, J. F., Swisdak, M., \& Reynolds, C. S. 2018b, ApJ, 867, 154, doi: $10.3847 / 1538-4357 /$ aae393

Ruszkowski, M., Brüggen, M., \& Begelman, M. C. 2004a, ApJ, 611, 158, doi: 10.1086/422158

—. 2004b, ApJ, 615, 675, doi: 10.1086/424702

Ruszkowski, M., \& Oh, S. P. 2010, ApJ, 713, 1332, doi: 10.1088/0004-637X/713/2/1332

Ryu, D., Kang, H., Hallman, E., \& Jones, T. W. 2003, ApJ, 593, 599, doi: 10.1086/376723

Sarazin, C. L. 1986, Reviews of Modern Physics, 58, 1, doi: 10.1103/RevModPhys.58.1

Schaal, K., Springel, V., Pakmor, R., et al. 2016, MNRAS, 461, 4441, doi: 10.1093/mnras/stw1587

Schekochihin, A. A., Cowley, S. C., Kulsrud, R. M., Hammett, G. W., \& Sharma, P. 2005, ApJ, 629, 139, doi: 10.1086/431202
Schlickeiser, R. 1989, ApJ, 336, 243 , doi: 10.1086/167009

Schlickeiser, R., \& Miller, J. A. 1998, ApJ, 492, 352, doi: $10.1086 / 305023$

Schwarzschild, K. 1906, Nachrichten von der Gesellschaft der Wissenschaften zu Göttingen, Mathematisch-Physikalische Klasse, 1906, 41. http://eudml.org/doc/58631

Sharma, P., Chandran, B. D. G., Quataert, E., \& Parrish, I. J. 2009, ApJ, 699, 348, doi: 10.1088/0004-637X/699/1/348

Skeel, R., \& Berzins, M. 1990, Siam Journal on Scientific and Statistical Computing, 11, doi: $10.1137 / 0911001$

Spitzer, L. 1956, Physics of Fully Ionized Gases Stewart, G. C., Canizares, C. R., Fabian, A. C., \& Nulsen, P. E. J. 1984, ApJ, 278, 536, doi: $10.1086 / 161820$

Thomas, T., \& Pfrommer, C. 2019, MNRAS, 485, 2977, doi: 10.1093/mnras/stz263

Tong, Y., Bale, S. D., Salem, C., \& Pulupa, M. 2018, arXiv e-prints. https://arxiv.org/abs/1801.07694

Tong, Y., Vasko, I. Y., Artemyev, A. V., Bale, S. D., \& Mozer, F. S. 2019, ApJ, 878, 41, doi: 10.3847/1538-4357/ab1f05

Vasko, I. Y., Krasnoselskikh, V., Tong, Y., et al. 2019, ApJ Lett., 871, L29, doi: 10.3847/2041-8213/ab01bd

Voigt, L. M., \& Fabian, A. C. 2004, MNRAS, 347, 1130, doi: 10.1111/j.1365-2966.2004.07285.x 
Xu, R., \& Kunz, M. W. 2016, Journal of Plasma Physics, 82, 905820507, doi: 10.1017/S0022377816000908

Yang, H. Y. K., \& Reynolds, C. S. 2016, APJ, 818, 181, doi: 10.3847/0004-637X/818/2/181

Zakamska, N. L., \& Narayan, R. 2003, ApJ, 582, 162, doi: 10.1086/344641
Zhuravleva, I., Churazov, E., Schekochihin, A. A., et al. 2014, Nature, 515, 85, doi: 10.1038/nature13830

Zweibel, E. G. 2013, Phys. Plasmas, 20, 055501, doi: $10.1063 / 1.4807033$

Zweibel, E. G., Mirnov, V. V., Ruszkowski, M., et al. 2018, ApJ, 858, 5, doi: 10.3847/1538-4357/aab9ae 

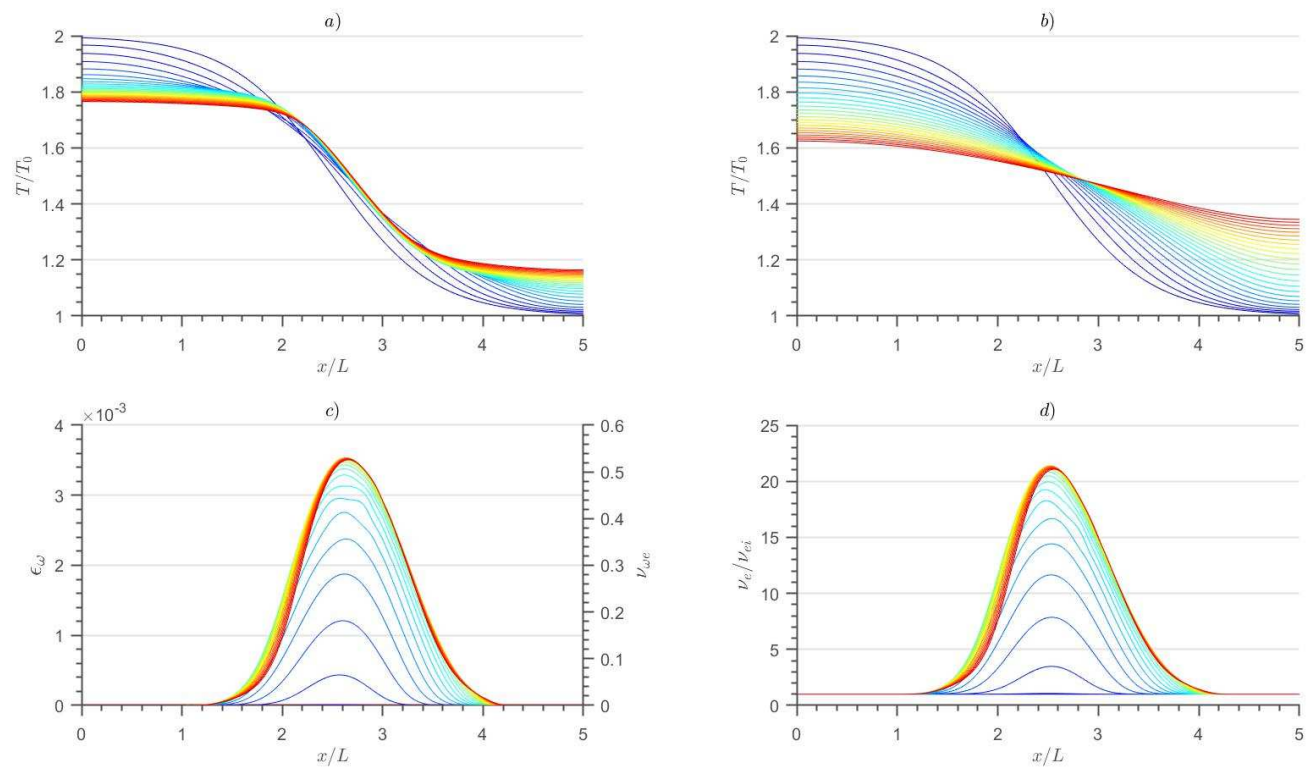

Figure 1. Results of a simulation of electron thermal transport in a regime with unstable whistlers $\left(L / \beta_{e} L_{c}=0.05\right)$. At several times $(t \in(0,0.15))$ during the growth phase of whistlers, cuts of the electron temperature $T$ with whistler scattering included (a) and with whistler scattering eliminated (b). From the simulation in (a) cuts of the energy density of rightward propagating whistlers $\varepsilon_{w}^{+}$and the associated whistler scattering rate $\nu_{w e}^{+}$in (c) and the ratio of the total electron scattering rate $\nu_{e}=\nu_{e i}+\nu_{w e}^{+}$to the electron-ion scattering rate $\nu_{e i}$ in $(\mathrm{d})$. 

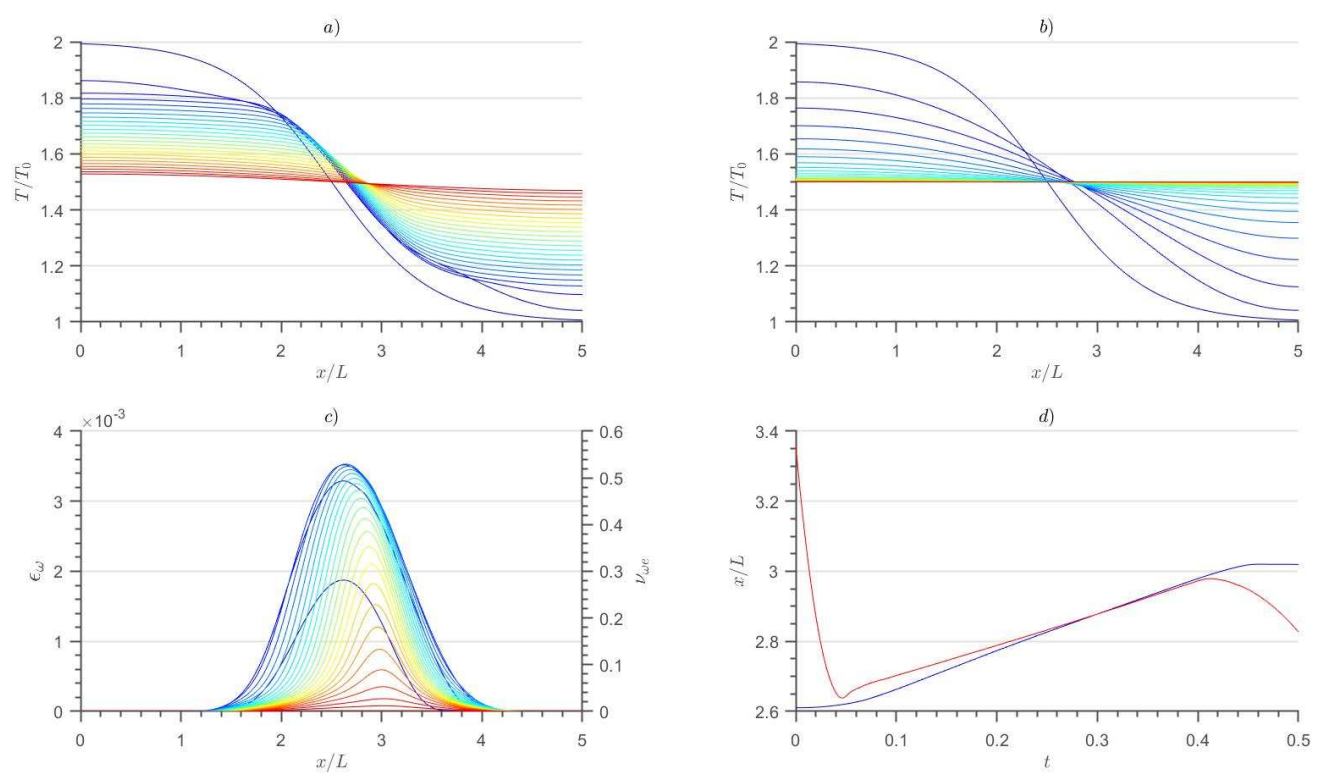

Figure 2. Results of the simulation of Fig. 1 over a longer time interval $(t \in(0,1.5))$. Cuts in (a), (b) and (c) as in Fig. 1. In (d) the trajectory of the location of the peak of $\varepsilon_{w}^{+}$(blue) and the maximum temperature gradient (orange). 

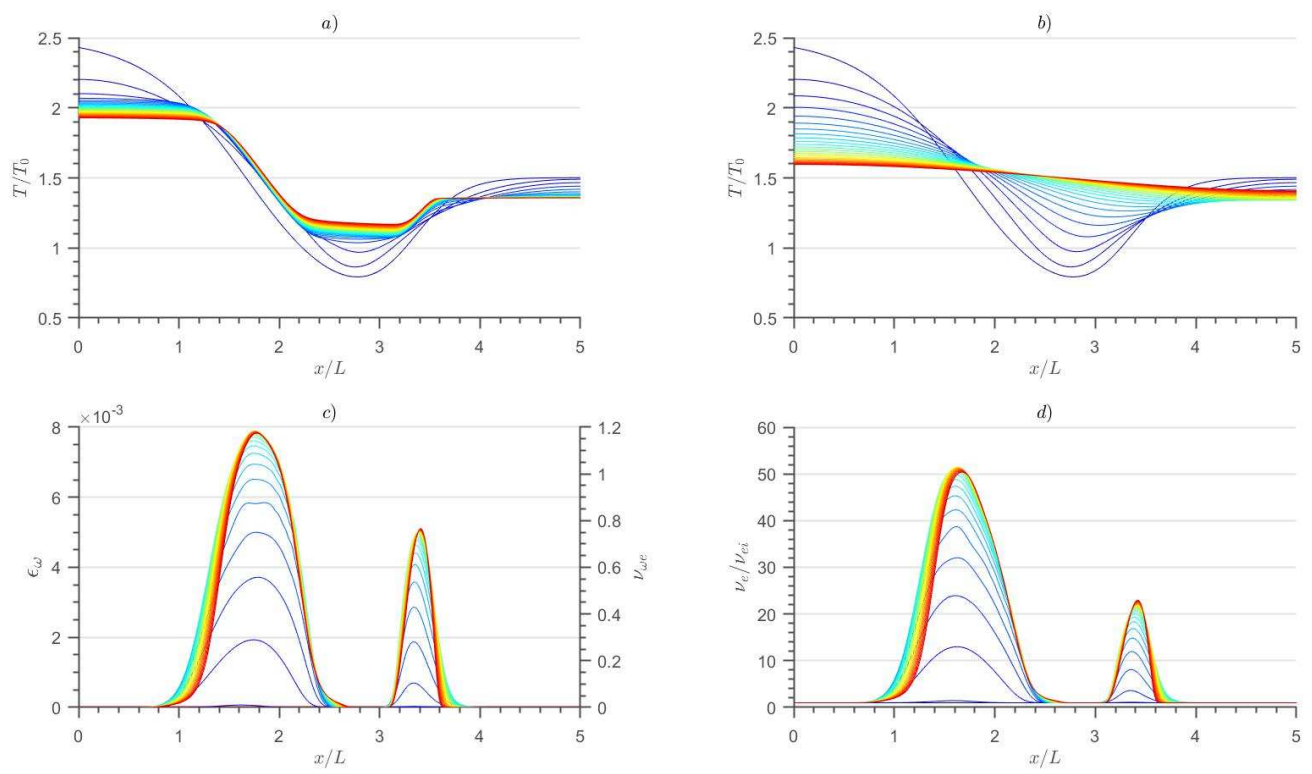

Figure 3. Results of a simulation of electron thermal transport in a system with a bi-directional temperature gradient and unstable whistlers propagating in the positive and negative directions. At several times $(t \in$ $(0,0.1))$ during the growth phase of whistlers, cuts of the electron temperature $T$ with whistler scattering included in (a) and with whistler scattering eliminated in (b). From the simulation in (a) cuts of the energy density of rightward, $\varepsilon_{w}^{+}$, and leftward $\varepsilon_{w}^{-}$, propagating whistlers and the associated whistler scattering rates $\nu_{w e}^{ \pm}$in (c). In (d) the ratio of the total electron scattering rate $\nu_{e}=\nu_{e i}+\nu_{w e}^{+}+\nu_{w e}^{-}$to the electron-ion scattering rate $\nu_{e i}$. 

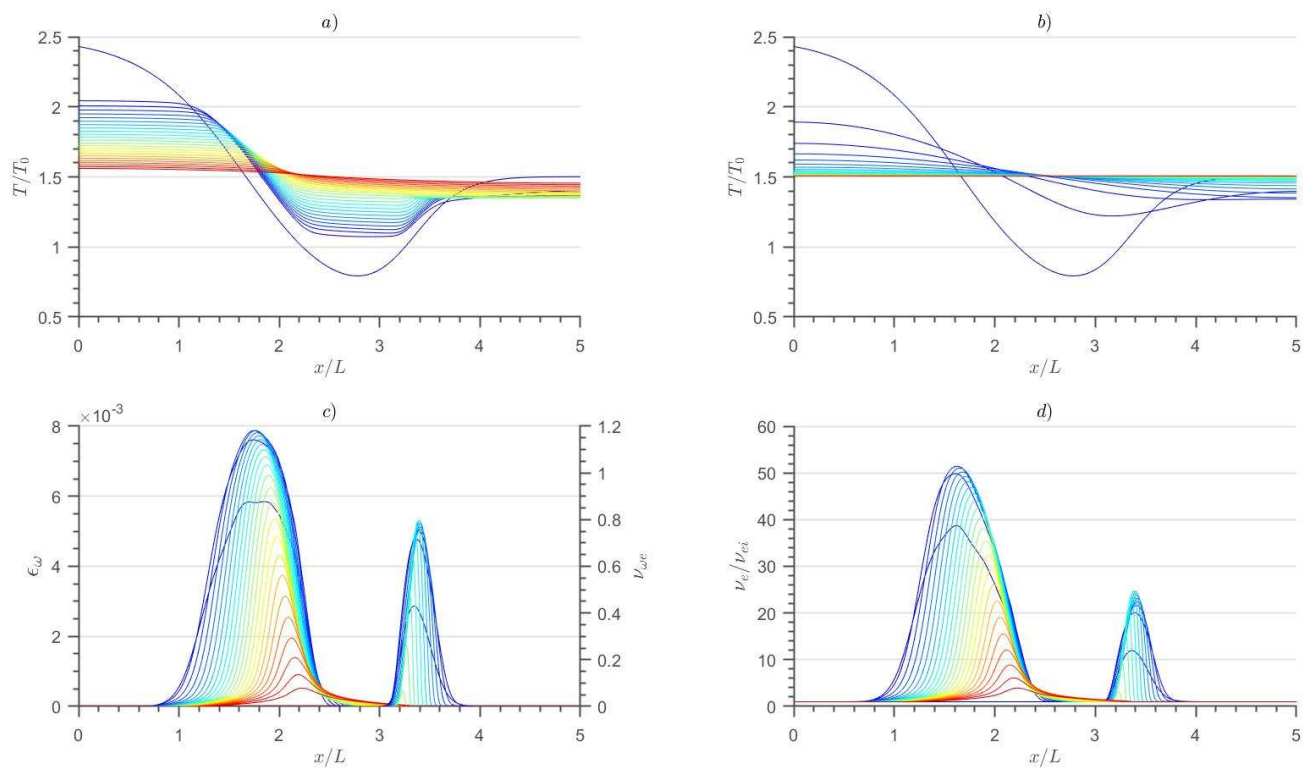

Figure 4. Results of the simulation of Fig. 3 over a longer time interval $(t \in(0,0.5))$. Cuts in (a), (b), (c) and $(\mathrm{d})$ as in Fig. 3.
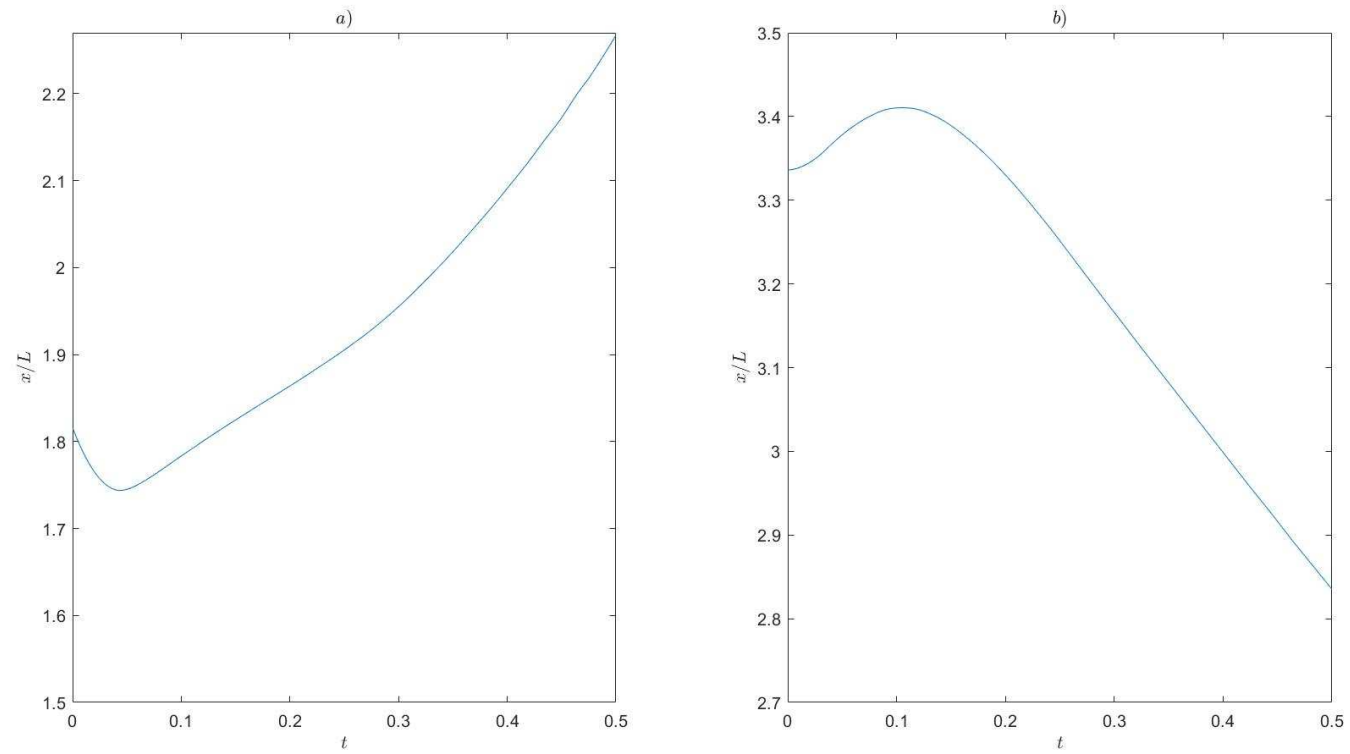

Figure 5. Results from the simulation of Fig. 4, the trajectories of the peaks of the wave energy of the rightward (a) and leftward (b) propagating whistlers. 